\title{
Simplicial Complex based Point Correspondence between Images warped onto Manifolds
}

\author{
Charu Sharma and Manohar Kaul \\ Indian Institute of Technology Hyderabad, India \\ \{cs16resch11007, mkaul\} @ith.ac.in
}

\begin{abstract}
Recent increase in the availability of warped images projected onto a manifold (e.g., omnidirectional spherical images), coupled with the success of higher-order assignment methods, has sparked an interest in the search for improved higher-order matching algorithms on warped images due to projection. Although currently, several existing methods "flatten" such 3D images to use planar graph / hypergraph matching methods, they still suffer from severe distortions and other undesired artifacts, which result in inaccurate matching. Alternatively, current planar methods cannot be trivially extended to effectively match points on images warped onto manifolds. Hence, matching on these warped images persists as a formidable challenge. In this paper, we pose the assignment problem as finding a bijective map between two graph induced simplicial complexes, which are higherorder analogues of graphs. We propose a constrained quadratic assignment problem (QAP) that matches each $p$-skeleton of the simplicial complexes, iterating from the highest to the lowest dimension. The accuracy and robustness of our approach are illustrated on both synthetic and real-world spherical / warped (projected) images with known ground-truth correspondences. We significantly outperform existing state-of-the-art spherical matching methods on a diverse set of datasets.
\end{abstract}

Keywords: Omnidirectional images, matching, assignment problem, QAP, simplicial complex

\section{Introduction}

There exists a longstanding line of research on finding bijective correspondences (i.e., assignments / matching $\{$, between two sets of visual features. Notable applications include stereo matching [14], structure from motion (SfM) [35], and image registration [32], to name a few. Traditionally, when matching points between multiple images of a fixed environment from various viewpoints, most approaches recover matchings and relative camera geometry (e.g. fundamental matrix) using a robust technique such as RANSAC [16]. On the other hand, when matching between different instances of the same category, graph matching methods [39] using unary and pairwise constraints have been successfully utilized. More recently, graph matching has been subsumed by hypergraph matching using higher-order constraints [12]19]. An important appeal of higher-order matching methods is their ability to coherently match compact local geometric features from the source space to similar compact regions in the target space,

1 assignment and matching are used interchangeably in this paper. 
despite the presence of noise, outliers, and incomplete data, thus achieving accurate matches that are also local structure-preserving in nature.

The recent proliferation of spherical images (e.g., omnidirectional and panoramic images captured from cameras mounted on drones and autonomous vehicles) and more generally, images warped onto manifolds with non-trivial curvatures, has sparked a heightened interest in assignment algorithms on such datasets due to the challenges they present in terms of curvature, both uniform and non-uniform [33|18|37|36]. Although assignment problems have been well studied for decades in computer vision, a majority of the work has only focused on matching points between planar (flat) images. Therefore, matching points on images with warping transformations which fall into the category of projective parametric models remains a challenging task, mainly due to the introduction of undesirable artifacts like severe distortions in pairwise distances between landmark points, non-linear distortions in local geometries, noise, illumination, blur, and occlusions [3], on flattening.

When dealing with matchings on curved geometries, primarily two types of methods are employed. Some putative matchings are computed to estimate a fundamental matrix [16[10] that captures the epipolar geometry of the 3D image. Stereo rectification [5] uses this fundamental matrix to re-project the two images on the same flat plane with row images aligned in parallel, followed by a re-matching to improve matching accuracy. Alternatively, geometric alignment on the fundamental matrix is used to verify and distinguish inliers from outliers, so that outliers can be pruned post matching to further boost accuracy [35]. Elements warped on the curved manifold cannot be metrically sampled in such methods and hence severe distortions are introduced [7], which is also consistent with the findings in our empirical studies.

Applications An interesting and noteworthy application of higher-order matching on spherical images arises in the area of biomedical imaging, especially in retinal imaging using optical coherence tomography (OCT). To investigate a wider field of view, 3D fundus images of the eye are captured, matched, and "stitched" together to form an $O C T$ montage [23 26]. This matching operation must additionally preserve regions of interest such as the optic cup / disc, fovea, macula, vessels, and microaneurysms, to name a few [30]. In addition to the standard noise, occlusion, and artifacts in these OCT fundus images, the data also suffers from data shifts due to axial eye motions and unpredictability between eye positions and instrument alignment across various scans [23]. Therefore, OCT datasets cannot easily be matched using rigid 3D transformations. Such images are not limited to merely spherical ones, but also arise in more general warped images due to projection. For instance, 3D sonograms depict the cervix as a conic frustum (truncated cone) [1] and clustered nanofluid microflow patterns in elastic micro-tubes are tracked via matching between cylindrical images in a time-lapse [34].

Related work Previous works on spherical matching mainly focus on producing good feature descriptors and can broadly be classified as planar and spherical feature extractors. Planar feature extractors like SIFT [24], SURF [4], ORB [28], BRISK [21], and FREAK [2] extract descriptors either on an unwrapped equirectangular version of an omnidirectional image or directly on the 2D (flat) representation of the spherical image. In contrast, spherical feature extractors reduce the distortion due to planar embeddings, by taking into account the underlying geodesic distances on the sphere while computing 
descriptors. These methods include ones based on spectral analysis, spherical harmonics (SIFTS [9]), and projection on geodesic grids (SPHORB [38], BRISKS [15]).

Our method In this paper, we focus on exploiting the intrinsic higher-order geometric relationships between landmark points on images warped onto curved manifolds. We capture these higher-order connections by constructing a combinatorial topological structure (simplicial complex) which is induced by a graph, whose vertices are the landmark points embedded on the warped image and whose edges are geodesic curves between selected vertex pairs. Next, we pose the assignment problem as a multi-dimensional quadratic assignment problem $(Q A P)$ between two graph-induced simplicial complexes.

Our contributions (i) To the best of our knowledge, we are the first to propose higher-order matching of landmark points on warped images projected onto curved manifolds (including for example spheres, ellipsoids, cylinders, and cones). (ii) In an attempt to break away from other works which solely focus on flat or spherical images, we propose a novel graph induced simplicial complex that efficiently captures higher order structures in a succinct manner, considering the inherent properties of the underlying manifold on which the landmark points are embedded. (iii) We uniquely formulate the assignment problem as a multi-dimensional combinatorial matching between two graph induced simplicial complexes, propose a novel algorithm to solve it, and analyze the time-complexity of our algorithm. (iv) Finally, to illustrate the robustness of our proposed method, we perform extensive experiments by comparing to planar matching methods, both existing and extended by us as naive baselines for matching of landmarks on manifolds. We compare our method against existing graph matching and spherical matching (both boosted using rectification and verification techniques) [21|28|38|39|40|31|12] on warped images and interestingly observe that not only does our method significantly outperform these matching methods on warped images onto curved manifolds (with up to $49.7 \%$ matching error reduction), but it also outperforms existing planar matching algorithms on "flat" planar images too (with up to $42.2 \%$ matching error reduction), due to the ability to naturally capture higher-order relationships by the simplicial complex.

\section{Preliminaries}

In this section, we introduce our notation and provide the necessary background for our higher-order assignment algorithm on curved manifolds. We begin by introducing certain standard definitions followed by our problem definition.

Let $\mathcal{M}$ denote a curved manifold. On a plane, the shortest distance between any two points is a straight line, i.e., a curve whose derivative to its tangent vectors is zero. We extend this notion of a "straight line" to curved manifolds by defining the shortest path (on $\mathcal{M}$ ) between its endpoints $u$ and $v$ placed on $\mathcal{M}$, as a geodesic curve $\gamma(u, v)$.

Simplicial complex We begin by providing some general definitions before we can formally define a simplicial complex. More background can be found in [25].

Given a set $V=\left\{v_{0}, \ldots, v_{n}\right\}$ of $(n+1)$ affinely independent points in $\mathbb{R}^{n+1}$, a $n$-dimensional simplex (also called $n$-simplex) $\sigma^{(n)}$ with vertices $V$ is the convex hull of 
$V$, i.e., more formally

$$
\sigma^{(n)}=\left\{\left(k_{0}, k_{1}, \ldots, k_{n}\right) \in \mathbb{R}^{n+1} \mid \sum_{i=0}^{n} k_{i}=1, k_{i} \geq 0 \forall i\right\}
$$

The dimension of $n$-simplex $\sigma^{(n)}$ is denoted by $\operatorname{dim}\left(\sigma^{(n)}\right)$. For example, a point/vertex (0-simplex), an edge (1-simplex), and a triangle (2-simplex) are represented as $\sigma^{(0)}$, $\sigma^{(1)}$, and $\sigma^{(2)}$, respectively.

For $0 \leq i \leq n$, the $i$-th facet $f_{i}$ of the $n$-simplex $\sigma^{(n)}$ is the $(n-1)$-simplex $\sigma^{(n-1)}$, whose vertices are those underlying $\sigma^{(n)}$, except the $i$-th vertex. For example, a 2 -simplex (triangle) has three 1-simplices (edges) as facets. The boundary $\partial \sigma^{(n)}$ of the $n$-simplex $\sigma^{(n)}$ is $\bigcup_{i=0}^{n} f_{i}$.

Finally, a simplicial complex $\mathcal{K}$ is a set of simplices that satisfy the following conditions:

(i). Any face of a simplex in $\mathcal{K}$ is a simplex in $\mathcal{K}$ and (ii). Intersection of distinct simplices $\sigma_{i}$ and $\sigma_{j}$ in $\mathcal{K}$, is a common face of both $\sigma_{i}$ and $\sigma_{j}{ }_{j}^{2}$

The $p$-skeleton $\mathcal{K}^{(p)} \subset \mathcal{K}$ is formed by the set of $k$-simplices $\sigma^{(k)}$, where $k \leq p$. Additionally, we denote by $\mathcal{K}_{k}$ the set of $k$-simplices in $\mathcal{K}$. The dimension $\operatorname{dim}(\mathcal{K})$ of a simplicial complex $\mathcal{K}$ is the maximum of the dimensions of its constituent simplices.

Problem definition Our problem consists of first constructing geometric simplicial complexes between landmark points given on curved manifolds, followed by finding an optimal (i.e., least cost) assignment between a pair of such geometric simplicial complexes by matching simplices of the same dimension, one dimension at a time.

More formally, Let $P$ and $P^{\prime}$ denote two sets of landmark points on curved manifolds $\mathcal{M}$ and $\mathcal{M}^{\prime}$, respectively. We construct geometric simplicial complexes $\mathcal{K}$ and $\mathcal{K}^{\prime}$ whose set of vertices ( 0 -simplices) are $P$ and $P^{\prime}$. The edges/arcs (1-simplices) in $\mathcal{K}$ and $\mathcal{K}^{\prime}$ are given by geodesics between select few pairs of vertices, from their corresponding vertex sets.

Given two simplicial complexes $\mathcal{K}$ and $\mathcal{K}^{\prime}$, we assume without loss of generality, that the number of simplices of each corresponding dimension are equal in both complexes. Then, our goal is to find a set of $h$ bijective matching functions $\left\{m_{k}\right\}_{k=0}^{h}: \mathcal{K} \longrightarrow \mathcal{K}^{\prime}$ that match the set of $k$-simplices in $\mathcal{K}$ (i.e., $\mathcal{K}_{k}$ ) to $k$-simplices in $\mathcal{K}^{\prime}$ (i.e., $\mathcal{K}_{k}^{\prime}$ ), for dimensions $k=0 \ldots h$, to minimize the overall objective function

$$
\underset{m_{0}, \ldots, m_{h}}{\operatorname{argmin}} \sum_{k=0}^{h} \sum_{i=1}^{\left|\mathcal{K}_{k}\right|} c\left(\sigma_{i}^{(k)}, m_{k}\left(\sigma_{i}^{(k)}\right)\right)
$$

where $c(\cdot, \cdot)$ is the geometric matching cost between a $k$-simplex $\sigma^{(k)}$ in $\mathcal{K}$ to a $k$-simplex $m\left(\sigma^{(k)}\right)$ in $\mathcal{K}^{\prime}$ and simplicial complex dimension $h=\min \left(\operatorname{dim}(\mathcal{K}), \operatorname{dim}\left(\mathcal{K}^{\prime}\right)\right)$.

Unlike formulations proposed in graph matching methods [39], where only node and pairwise geometric relations are considered, our combinatorial optimization formulation takes into consideration higher-order geometric constraints, which better excludes ambiguous matchings.

${ }^{2}$ For ease of notation, we drop the dimension superscript and index subscript for a simplex when it is understood from context. 
In subsequent sections, we show how we construct such geometric simplicial complexes from the landmark points on curved manifolds (Section 3), followed by a detailed explanation of our assignment algorithm (Section 4 ).

\section{Building a Simplicial Complex on a Curved Manifold}

In this section, inspired by the work of Dey et. al. [11], we similarly construct a graphinduced simplicial complex, which is built upon a graph connecting the landmark points. We begin by describing the process of constructing the underlying graph.

Graph construction Let $(P, g)$ denote the set of landmark points $P$ with a metric $g$ that denotes the geodesic distance between a pair of points on $\mathcal{M}$. Additionally, let the $k$-neighborhood $\mathcal{N}_{k}(u)$ denote the set of $k$ nearest neighbors of landmark point $u \in P$ (inclusive of $u$ ) on manifold $\mathcal{M}$ according to the geodesic metric $g$.

Considering all ordered pairs $(u, v)$, where $u, v \in P$, an undirected edge/arc is introduced between points $u$ and $v$, when their corresponding $k$-neighborhoods $\mathcal{N}_{k}(u)$ and $\mathcal{N}_{k}(v)$ have a non-empty intersection, i.e., $\mathcal{N}_{k}(u) \cap \mathcal{N}_{k}(v) \neq \emptyset$. All such edges are collected into a set denoted by $E$. This completes the construction of our underlying graph $G=(P, E)$. Observe that the vertex set (landmarks) $P$ form the 0 -skeleton $\mathcal{K}^{(0)}(G)$ and the sets $E$ and $P$ together form the 1 -skeleton $\mathcal{K}^{(1)}(G)$, of our graphinduced simplicial complex that we will denote by $\mathcal{K}(G)$.

Recall that a $n$-clique in a graph is a complete subgraph between $n$ vertices, i.e., it consists of $n$ vertices and $\left(\begin{array}{l}n \\ 2\end{array}\right)$ edges.

Graph-induced complex $\mathcal{K}(G)$ is defined as the simplicial complex where a $n$ simplex $\sigma^{(n)}=\left\{p_{1}, p_{2}, \ldots, p_{n+1}\right\}$ is in $\mathcal{K}(G)$, if and only if there exists a $(n+1)$-clique $\left\{p_{1}, p_{2}, \ldots, p_{n+1}\right\} \subseteq P$ in the underlying graph $G=(P, E)$. In words, the cliques of the underlying graph $G=(P, E)$ form the simplices in $\mathcal{K}(G)$ because cliques satisfy both conditions of being a simplicial complex (which can be trivially verified). In order to be used in our assignment algorithm, we must represent the graph-induced simplicial complex $\mathcal{K}(G)$ as a set of boundary matrices, which we present next.

Matrix representation of $\mathcal{K}(G)$ : Given $\mathcal{K}(G)$ and its $p$-skeleton $\mathcal{K}^{(p)}(G)$ that contains cliques upto size $p+1$, we represent it as a boundary matrix $M_{p} \in \mathbb{Z}^{n \times m}$ defined as

$$
M_{p}=\underset{\tau_{n}^{(p-1)}}{\tau_{n}^{(p-1)}}\left(\begin{array}{ccc}
\sigma_{1}^{(p)} & \ldots & \sigma_{m}^{(p)} \\
a_{11} & \ldots & a_{1 m} \\
\vdots & \ddots & \vdots \\
a_{n 1} & \ldots & a_{n m}
\end{array}\right)
$$

where $a_{i j}=1$ if and only if the $i$-th $(p-1)$-simplex $\tau_{i}^{(p-1)}$ is a facet of the $j$-th $p$-simplex $\sigma_{j}^{(p)}$, otherwise $a_{i j}=0$. Then, the boundary of a $j$-th $p$-simplex is given by $\partial_{p} \sigma_{j}^{(p)}=\sum_{i=1}^{n} a_{i j} \tau_{i}^{(p-1)}$.

Observe that the $p$-th boundary matrix $M_{p}$ captures all possible relationships between $p$-simplices and their $(p-1)$-simplex boundaries (or facets). Boundary matrix $M_{p}$ is made for each $p$-skeleton and therefore $\mathcal{K}(G)$ is expressed as a set of boundary matrices $\left\{M_{p}\right\}_{p=1}^{h}$, where $h=\operatorname{dim}(\mathcal{K}(G))$. 
Remark 1. Our underlying graph $G$ already contains as a subgraph a simple $k$-nearest neighbor graph which is constructed by introducing edges between a vertex in question and its $k$ nearest neighbors. Therefore, our underlying graph $G$ has more edges and thus has a higher likelihood to form higher-order relations between vertices. On the other hand, while the Delaunay triangulation is simple to compute and is a good vehicle for extracting topology of sampled spaces, its size becomes prohibitively large for reasonable computations and thus adversely affects the QAP matching algorithm.

In summary, our underlying graph $G$ which is inspired by the Vietoris-Rips complex construction provides a good proximity structure, which is neither too sparse (like simple $k$-NN graphs) or too dense (like Delaunay triangulated graphs) and encodes useful higher-order information about local relations of landmark points in $P$.

\section{Assignment Algorithm}

Recall our problem definition (Section 2) of trying to find a set of assignments / matching functions between two graph-induced simplicial complexes $\mathcal{K}(G)$ and $\mathcal{K}\left(G^{\prime}\right)$. Here, we outline the details of our assignment algorithm.

Given a boundary matrix $M_{p} \in \mathbb{Z}^{n \times m}$ that represents a $p$-skeleton $\mathcal{K}^{(p)}(G)$, we first capture the geodesic neighborhood geometry of simplices in $M_{p}$. We begin by defining an adjacency operator $\sim$ between two simplices followed by a definition of a neighborhood of a simplex. This neighborhood of a simplex is then elegantly captured by affine weight vectors, which are later used in the matching algorithm.

Definition 1 (adjacency relation). Given two simplices $\sigma^{(d)}$ and $\sigma^{\prime\left(d^{\prime}\right)}$, each of arbitrary dimension $d$ and $d^{\prime}$, we consider them to be adjacent to one another if and only if they share a common simplex. We denote this adjacency relation by $\sigma^{(d)} \sim \sigma^{\prime\left(d^{\prime}\right)}$. The dimension of the common simplex can take values from 0 to $\min \left(d, d^{\prime}\right)$.

For example, two 2-simplices / triangles $\sigma^{(2)}$ and $\sigma^{\prime(2)}$ could either be connected at a common 0 -simplex / vertex or share a common 1-simplex / edge; both cases would result in the simplices being adjacent, i.e., $\sigma^{(2)} \sim \sigma^{\prime(2)}$.

Simplex neighborhood The boundary matrix $M_{p}$ 's columns encode $p$-simplices $\sigma_{1}^{(p)}, \ldots, \sigma_{m}^{(p)}$ and its rows encode $(p-1)$-simplices $\tau_{1}^{(p-1)}, \ldots, \tau_{n}^{(p-1)}$. The computation of the neighborhood $\mathfrak{N}(\cdot)$ for $p$-simplices and $(p-1)$-simplices differ slightly. The neighborhood of a $p$-simplex consists of $p$-simplices (same dimension) and $(p-1)$ simplices (one dimension lower) that are adjacent to it. While, the neighborhood of a $(p-1)$-simplex consists of $(p-1)$-simplices (same dimension) and $p$-simplices (one dimension higher) that are adjacent to it. More formally, the neighborhood of the $i$-th $p$-simplex $\sigma_{i}^{(p)}$ is

$$
\mathfrak{N}\left(\sigma_{i}^{(p)}\right)=\left\{\sigma_{j}^{(p)} \mid \sigma_{j}^{(p)} \sim \sigma_{i}^{(p)}\right\} \cup\left\{\tau_{j}^{(p-1)} \mid \tau_{j}^{(p-1)} \sim \sigma_{i}^{(p)}\right\}
$$

and the neighborhood of the $i$-th $(p-1)$-simplex $\tau_{i}^{(p-1)}$ is

$$
\begin{aligned}
\mathfrak{N}\left(\tau_{i}^{(p-1)}\right)= & \left\{\tau_{j}^{(p-1)} \mid \tau_{j}^{(p-1)} \sim \tau_{i}^{(p-1)}\right\} \\
& \cup\left\{\sigma_{j}^{(p)} \mid \sigma_{j}^{(p)} \sim \tau_{i}^{(p-1)}\right\}
\end{aligned}
$$


Such neighborhoods are computed for all the $p$ - and $(p-1)$-simplices in $M_{p}$, where $i \neq j$.

Affine weight vectors For a $p$-simplex $\sigma^{(p)}$, let $\mathcal{B}\left(\sigma^{(p)}\right)$ denote the set of all the barycenters $\left\{b_{1}, \ldots, b_{\left|\mathfrak{N}\left(\sigma^{(p)}\right)\right|}\right\}$ of the simplices in the neighborhood $\mathfrak{N}\left(\sigma^{(p)}\right)$. Then, $\sigma^{(p)}$ is represented as an affine combination of the barycenters in $\mathcal{B}\left(\sigma^{(p)}\right)$, i.e., $\sum_{i=1}^{\left|\mathcal{B}\left(\sigma^{(p)}\right)\right|} \alpha_{i} b_{i}$, where $\sum_{i=1}^{\left|\mathcal{B}\left(\sigma^{(p)}\right)\right|} \alpha_{i}=1$ (i.e., weights $\alpha_{i}$ 's must sum to 1 ). Therefore, $\sigma^{(p)}$ is expressed as an affine weight vector $\alpha\left(\sigma^{(p)}\right)$ of dimension $(n+m)$, with $\left|\mathcal{B}\left(\sigma^{(p)}\right)\right|$ positions corresponding to $\mathfrak{N}\left(\sigma^{(p)}\right)$ filled with non-empty affine weights and the rest set to zero. Such an affine weight vector is computed for every simplex of dimension $p$ and $(p-1)$ contained in $M_{p}$.

Following a similar method as shown in [22] for the choice of a locally invariant affine weight vector, we extend their method to simplices and among all possible affine representations of a simplex, we chose to use least squares to guarantee minimal error under L2-norm, and furthermore it assigns non-zero weights to each of its adjacent simplex barycenters, thereby better capturing the local geometric properties in its neighborhood.

Remark 2. The affine weight vectors act as locally affine invariant descriptors that can handle complex and natural transformations of the underlying manifold $\mathcal{M}$. Additionally, it allows for much fewer variables and can be much more easily linearized in the subsequent QAP formulation. Furthermore, the inclusion of barycenters from neighborhoods of each simplex act as higher-order geometric constraints that easily excludes ambiguous matchings. In comparison, simple matching models that rely on just a distance matrix with pairwise geodesic distances on the manifold are not invariant to local and global affine transformations and completely disregard higher-order relationships.

Cost matrix construction Next, we describe the construction of a cost matrix that is needed to compute assignments between $M_{p} \in \mathbb{Z}^{n \times m}$ and $M_{p}^{\prime} \in \mathbb{Z}^{n^{\prime} \times m^{\prime}}$. We begin by constructing two cost matrices $\mathcal{C}^{(p-1)} \in \mathbb{R}^{n \times n^{\prime}}$ and $\mathcal{C}^{(p)} \in \mathbb{R}^{m \times m^{\prime}}$ to measure the Euclidean distance between the affine weight vectors of $(p-1)$-simplices and the Euclidean distance between the affine weight vectors of $p$-simplices, respectively.

More specifically, $c_{i i^{\prime}}^{(p-1)}=\left\|\alpha\left(\tau_{i}^{(p-1)}\right)-\alpha\left(\tau_{i^{\prime}}^{(p-1)}\right)\right\|_{2}$, measures the Euclidean distance between the affine weight vectors of the $i$-th $(p-1)$-simplex of $M_{p}$ and the $i^{\prime}$-th $(p-1)$-simplex of $M_{p}^{\prime}$, while $c_{k k^{\prime}}^{(p)}=\left\|\alpha\left(\sigma_{k}^{(p)}\right)-\alpha\left(\sigma_{k^{\prime}}^{(p)}\right)\right\|_{2}$, measures the Euclidean distance between the affine weight vectors of the $k$-th $p$-simplex of $M_{p}$ and the $k^{\prime}$-th $p$-simplex of $M_{p}^{\prime}$.

Similar to the affinity matrix construction in [39], we combine both the cost matrices in a single geodesic-cost matrix $\mathcal{L}^{(p)}=\left(l_{i i^{\prime}, j j^{\prime}}\right) \in \mathbb{R}^{n n^{\prime} \times m m^{\prime}}$ as

$$
l_{i i^{\prime}, j j^{\prime}}^{(p)}= \begin{cases}c_{i i^{\prime}}^{(p-1)} & i=j, i^{\prime}=j^{\prime} \\ c_{k k^{\prime}}^{(p)} & i \neq j, i^{\prime} \neq j^{\prime}, a_{i k} a_{j k} a_{i^{\prime} k^{\prime}}^{\prime} a_{j^{\prime} k^{\prime}}^{\prime}=1 \\ 0 & \text { otherwise }\end{cases}
$$

The diagonal and off-diagonal entries of matrix $\mathcal{L}^{(p)}$ capture the Euclidean distances between the affine weight vectors of $(p-1)$-simplices and the Euclidean distances between the affine weight vectors of p-simplices, respectively. Therefore, our QAP can 


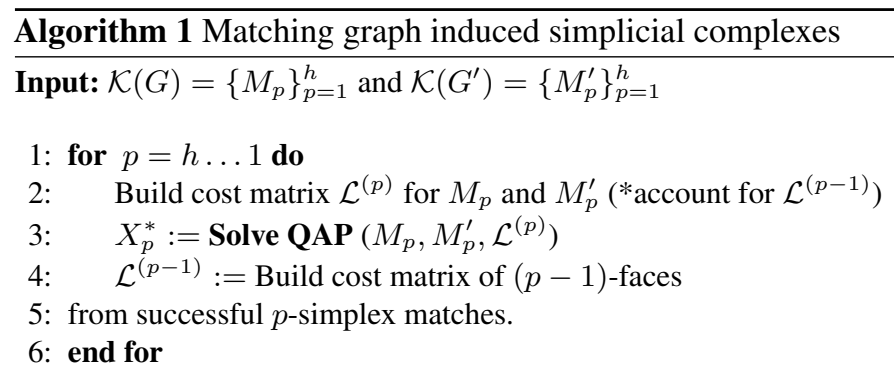

Return: $\left\{X_{1}^{*}, \ldots, X_{h}^{*}\right\}$ \# set of permutation matrices
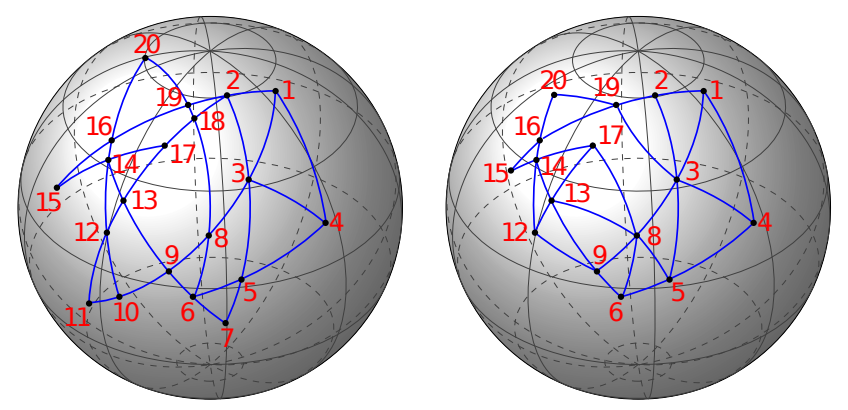

Fig. 1. Pair of spheres with simplicial complexes constructed between the landmark points on the spheres along with assignments between cliques.

now be formulated as

$$
\begin{array}{ll}
\underset{X_{1}, \ldots, X_{h}}{\operatorname{argmin}} & \sum_{p=1}^{h} \operatorname{vec}\left(X_{p}\right)^{T} \mathcal{L}^{(p)} \operatorname{vec}\left(X_{p}\right) \\
\text { subject to } & \forall p \leq h, \mathbb{1}^{T} X_{p}=\mathbb{1}, X_{p}^{T} \mathbb{1}=\mathbb{1}
\end{array}
$$

where $X_{p}$ is a permutation matrix and $\operatorname{vec}\left(X_{p}\right)$ is it's vector representation. Our solution to Equation 2 is concisely outlined in Algorithm 1 . As we solve a QAP from highest to lowest dimension $p$-skeleton, we track the $(p-1)$-simplices whose matchings are induced by higher order simplex matches. On finding $(p-1)$-simplices that have the lowest cost and cannot be improved by solving a lower level QAP, we eliminate such simplices, causing the size of the matrix to shrink in subsequent iterations, leading to substantial speedups. Also, we use a spectral relaxation proposed by Lordeneu et. al. [20] to solve our QAP efficiently.

Example We illustrate with an example the bijective assignment produced by our algorithm between cliques / simplices of a pair of graph-induced spherical simplicial complexes, as shown in Figure 1 . We consider two simplicial complexes $\mathcal{K}$ and $\mathcal{K}^{\prime}$ each 
Table 1. Matchings of 3, 2-cliques of simplicial complexes $\mathcal{K}$ and $\mathcal{K}^{\prime}$ shown in Figure 1

\begin{tabular}{cll}
\hline$k$-Clique & \multicolumn{3}{c}{ Matching between $\mathcal{K} \boldsymbol{\&} \mathcal{K}^{\prime}$} \\
\hline 3-Cliques & $(1,2,3),(1,3,4),(3,4,5)$, & $(1,2,3),(1,3,4),(3,4,5)$, \\
& $(2,18,19),(5,6,7),(6,8,9)$, & $(2,3,19),(5,6,8),(6,8,9)$, \\
& $(12,13,14),(13,14,17)$, & $(12,13,14),(13,14,17)$, \\
& $(14,15,16),(16,19,20)$. & $(14,15,16),(16,19,20)$. \\
\hline 2-Cliques & $(1,2),(1,3),(2,3),(1,4)$, & $(1,2),(1,3),(2,3),(1,4)$, \\
& $(3,4),(3,5),(4,5),(2,19)$, & $(3,4),(3,5),(4,5),(2,19)$, \\
& $(18,19),(5,6),(5,7),(6,8)$, & $(3,19),(5,6),(5,8),(6,8)$, \\
& $(6,9),(8,9),(12,13),(12,14)$, & $(6,9),(8,9),(12,13),(12,14)$, \\
& $(13,14),(13,17),(14,17)$, & $(13,14),(13,17),(14,17)$, \\
& $(14,15),(14,16),(15,16)$, & $(14,15),(14,16),(15,16)$, \\
& $(16,19),(16,20),(19,20)$, & $(16,19),(16,20),(19,20)$, \\
& $(3,8),(8,18),(9,10),(9,13)$, & $(3,8),(8,13),(9,12),(9,13)$, \\
& $(17,18)$. & $(17,8)$. \\
\hline
\end{tabular}

embedded on $\mathcal{S}^{2}$, with 20 and 16 vertices, respectively. Matching of corresponding 3cliques and 2-cliques are mentioned in the Table 1 . Matching between vertices (1-cliques) is shown by marking them with the same label on both spheres.

Time complexity analysis The major cost incurred by our algorithm arises from matching cliques between two simplicial complexes. Therefore, we first derive an upper bound on the number of cliques that need to be matched as follows (proof in supplementary notes).

Lemma 1. Let $\mathcal{K}(G)$ represent the simplicial complex induced from graph $G$ with $n$ and $m$ number of vertices and edges, respectively. Let $h$ denote the maximum order of cliques in $G$ and $\delta$ be the maximum degree of a vertex in $G$. Then, the total number of $k$-cliques in $\mathcal{K}(G)$ for $k=(1, \ldots, h)$, are at most

$$
n+\frac{2 m}{\delta(\delta+1)}\left[\min \left\{(\delta+1)^{h}+1,\left(\frac{e(\delta+1)}{h}\right)^{h}\right\}-\delta-2\right]
$$

Neglecting lower order terms, the number of cliques are of order $O\left(n+m\left(\delta^{h-2}-\delta\right)\right)$. We know that the spectral relaxation proposed by Lordeneu et. al. [20] has a complexity of $O\left(n^{3 / 2}\right)$, where $n$ is the number of points to match on each side. Our higher order matching of cliques then has a time complexity of $O\left(\left\{n+m\left(\delta^{h-2}-\delta\right)\right\}^{3 / 2}\right)$. In practice, for maximum order of cliques, $h=3$ (triangles) and $h=4$ (tetrahedrons), observe that the complexity drops to $O\left(n^{3 / 2}\right)$ and $O\left(\left\{n+m \delta^{2}\right\}^{3 / 2}\right)$, respectively, which is very efficient.

\section{Experiments}

For our experiments, we considered synthetic and real-world datasets that cover both spherical and planar images. Spherical images can broadly be categorized as: parabolic omnidirectional $\left(360^{\circ}\right)$, fish-eye, and panoramic images. Note that our matching algorithm does not require any calibration parameters of cameras.

To evaluate our matching algorithm, we compared against three main categories. (i) Planar matching methods extended with geodesic metric on $3 D$ manifolds: Here, 
we extended the factorized graph matching (FGM) [39] algorithm by feeding it a $k$ $\mathrm{NN}$ graph based on geodesic distances between points to serve as our naive baseline method (called "FGM+geodesic"). The rest of the methods were feature-descriptor based. (ii) Planar matching methods on $2 D$ projected (unwrapped ${ }^{3}$ ) manifolds. (iii) Planar matching methods on $2 D$ planar images: Here, we proposed a flat version of our algorithm with Euclidean distance as the underlying metric (called "OurPlanar") to work on flat $2 \mathrm{D}$ images.

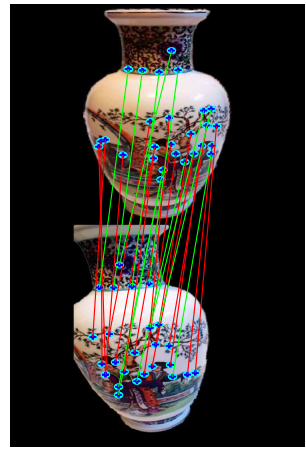

(a)

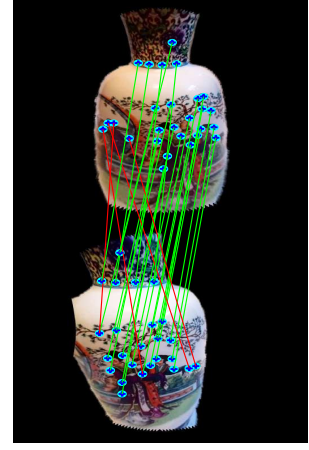

(b)

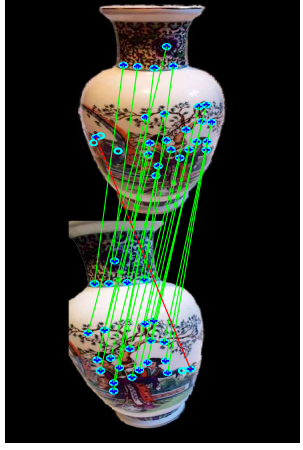

(c)

Fig. 2. Instances of matchings between (a) Chinese vase images for Tensor based method, (b) flat version of Chinese vase images for Tensor based method, and (c) Chinese vase images for our method. Green/red lines show correct/incorrect matches respectively. Isolated points show no matches.

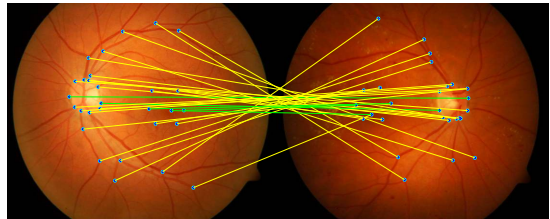

(a)

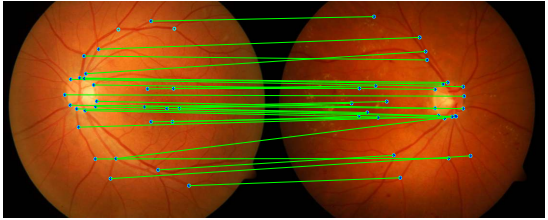

(b)

Fig. 3. Instances of matchings between (a) Fundus images for Tensor based method, (b) Fundus images for our method. Green/yellow lines show correct/incorrect matches respectively. Isolated points show no matches.

Furthermore, we conducted additional matching experiments on spherical/warpedplanar images and unwrapped spherical/warped-planar images whose results are shown in our supplementary material. We also perform experiments using RANSAC [16] for geometric verification and rectification. In our ablative studies, we analyze the robustness of our algorithm under affine transformations (rotation, reflection, scaling, and shear), under the effect of two noise models (in supplementary material) proposed in [13], and the effects of randomly removing some landmarks to simulate missing completely at random (MCAR) phenomena (in supplementary material). Additional experiments on

3 unwrapped implies planar projection of a spherical image with minimal distortion [9] [15]. 
rotation for SUN360 dataset and Desktop dataset and the effects of varying $k$ values in a neighborhood of landmark points, varying the radius of the sphere and manifold, and varying the number of landmark points for matching are also shown in supplementary material.

Baselines We group the state-of-the-art methods as: (i) Feature descriptor based matching for spherical and planar images: BRISK [21], ORB [28], SPHORB [38 ${ }^{4}$ (ii) Graph based matching for planar images: based on employing an affinity matrix (FGM) [3940 $]^{5}$ and eigenvalues (EigenAlign) [13 $]^{6}$ (iii) Higher-order based matching for planar images: using random clique complex (RCC) [31] and higher-order matching (Tensor) [12] (iv) Finally, a naive baseline (FGM+geodesic) proposed by us that extends FGM by constructing a graph based on geodesic distances.

Datasets We sourced raw spherical image datasets without landmarks and annotated them using FAST (Features from Accelerated Segment Test) corner detector [27] and generated the matching ground truth for all the datasets used in our experiments. All six datasets are as follows (with their details in supplementary): (i) Chessboard dataset consists of omnidirectional and fish-eye images. (ii) Kamaishi and (iii) SUN360 dataset has panoramic images. (iv) Desktop dataset consists of omnidirectional and planar images. $(v)$ Parking dataset also has omnidirectional and planar images. (vi) Table dataset contains omnidirectional, fish-eye, and planar images. Our code $\AA^{8}$ is publicly available.

Table 2. Error (\%) of pairwise matching between spherical images (omnidirectional, fish-eye and panorama) of five datasets for different methods.

\begin{tabular}{llllll}
\hline Algorithms & Kamaishi & Chessboard & Desktop & Parking & Table \\
\hline OurWarped & $\mathbf{0 . 7 9} \pm \mathbf{0 . 0} \%$ & $\mathbf{3 . 8 9} \pm \mathbf{0 . 0} \%$ & $\mathbf{0 . 3 2} \pm \mathbf{0 . 0} \%$ & $\mathbf{0 . 0} \pm \mathbf{0 . 0} \%$ & $\mathbf{0 . 7 4} \pm \mathbf{0 . 0} \%$ \\
\hline FGM+geo & $55.6 \pm 0.10 \%$ & $79.2 \pm 1.21 \%$ & $23.3 \pm 0.03 \%$ & $37.5 \pm 0.0 \%$ & $64.3 \pm 6.58 \%$ \\
SPHORB & $90.0 \pm 0.0 \%$ & $58.5 \pm 0.0 \%$ & $91.1 \pm 0.0 \%$ & $95.0 \pm 0.0 \%$ & $78.5 \pm 0.0 \%$ \\
BRISK & $85.6 \pm 0.0 \%$ & $53.6 \pm 0.0 \%$ & $78.9 \pm 0.0 \%$ & $81.6 \pm 0.0 \%$ & $69.2 \pm 0.0 \%$ \\
ORB & $90.2 \pm 0.0 \%$ & $53.8 \pm 0.0 \%$ & $51.7 \pm 0.0 \%$ & $71.1 \pm 0.0 \%$ & $64.4 \pm 0.0 \%$ \\
Tensor & $37.7 \pm 0.69 \%$ & $60.5 \pm 0.41 \%$ & $23.9 \pm 1.7 \%$ & $23.7 \pm 7.5 \%$ & $85.1 \pm 1.05 \%$ \\
FGM & $53.3 \pm 0.21 \%$ & $80.0 \pm 0.11 \%$ & $31.9 \pm 0.12 \%$ & $36.0 \pm 1.5 \%$ & $65.5 \pm 0.01 \%$ \\
\hline
\end{tabular}

Our method vs. planar matching methods with geodesic metric on 3D curved manifolds Here, we match pairwise images directly on the warped images on curved manifolds (as shown in Figures 2, 3, and 4(b)). The comparison between standard higherorder graph matching (Tensor) [12] and our method on manifold is shown in Figures 2 and 3 using Chinese vase ${ }^{9}$ and Fundus images [17], respectively. We observe from Figures 2(a) and 3(a) that the Tensor based method does not perform well on warped images. Although, the matching does improve when images are flattened to reduce the effect of curvature in Figure 2(b), Our method outperforms the baseline and has a maximum number of correct matches in Figures 2(c) and 3(b).

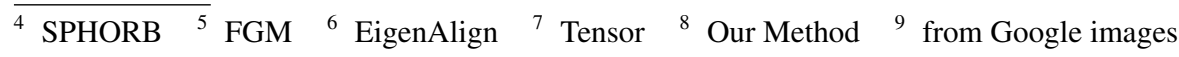


The error percentages of our warped image matching algorithm (OurWarped) are shown in the first row of Table 2 . We observe that our method outperforms all other matching methods, including spherical feature descriptor based ones as well. Additional multimodal warped-planar matching experiments can be found in our supplementary notes.

For matches between spherical and planar images, we find two variants which match between a spherical and a planar image (Figure 4(a) and matching between different types of spherical images (Figure 4(b)). In Table 2, there is a slight increase in error percentages when matching across different types of spherical images, i.e., $3.89 \%$ for Chessboard, as compared to matching similar types, i.e., $0.32 \%$ for Desktop, due to differences in distortion levels. In spite of this, we find that our method significantly outperforms naive baseline and other matching methods on spherical images.

Table 3. Error (\%) of pairwise matching between unwrapped equirectangular version of spherical (omnidirectional and fish-eye) images of four datasets for different methods including graph matching methods on flat surfaces.

\begin{tabular}{lllll}
\hline Algorithms Chessboard & Desktop & Parking & Table \\
\hline OurWarped & $\mathbf{3 . 6 4} \pm \mathbf{0 . 0} \%$ & $\mathbf{1 . 0 6} \pm \mathbf{0 . 0} \%$ & $\mathbf{0 . 0} \pm \mathbf{0 . 0} \%$ & $\mathbf{0 . 5 7} \pm \mathbf{0 . 0} \%$ \\
\hline RCC & $28.6 \pm 0.94 \%$ & $11.6 \pm 0.74 \%$ & $13.2 \pm 11.8 \%$ & $11.6 \pm 0.57 \%$ \\
EigenAlign & $98.47 \pm 0.0 \%$ & $95.24 \pm 0.0 \%$ & $97.5 \pm 0.0 \%$ & $97.9 \pm 0.0 \%$ \\
Tensor & $68.9 \pm 0.16 \%$ & $26.1 \pm 0.58 \%$ & $19.0 \pm 3.75 \%$ & $72.4 \pm 0.67 \%$ \\
FGM & $84.0 \pm 0.0 \%$ & $31.0 \pm 0.0 \%$ & $38.0 \pm 0.0 \%$ & $52.0 \pm 0.0 \%$ \\
SPHORB & $58.6 \pm 0.0 \%$ & $90.3 \pm 0.0 \%$ & $97.5 \pm 0.0 \%$ & $79.2 \pm 0.0 \%$ \\
BRISK & $54.9 \pm 0.0 \%$ & $84.9 \pm 0.0 \%$ & $100.0 \pm 0.0 \%$ & $74.2 \pm 0.0 \%$ \\
ORB & $49.5 \pm 0.0 \%$ & $78.2 \pm 0.0 \%$ & $82.5 \pm 0.0 \%$ & $70.3 \pm 0.0 \%$ \\
\hline
\end{tabular}

Our method vs. planar matching methods on 2D-projected curved manifolds Matching between spherical images can also be performed by applying planar graph matching methods on unwrapped equirectangular versions of spherical images. This makes the image flat and standard planar matching algorithms can then be employed. However, any kind of projection (on a flat surface in this case) introduces distortions in the resulting image. We flattened spherical images for four datasets mentioned in Table 3 . We used two different methods to flatten omnidirectional and fish-eye images. The $360^{\circ}$ image is unwrapped by dividing it into four parts (quadrants) and concatenated into a single flat image. On the other hand, fish-eye images do not cover the complete view of the scene and add distortion to the image due to curved mirrors and lenses of the cameras. We try to reduce the distortion by removing curves and flattening the image using calibration techniques outlined in [29]. Since any projection will lead to distortion, we can compare the results from Table 2 with Table 3 . Both the experimental outcomes are based on the same set of spherical images. Our matching algorithm significantly outperforms its competitors on both the spherical images and on curved manifolds.

Our method vs. planar matching methods on 2D planar images Our proposed method can also be reduced to a higher-order planar graph matching method. To show the importance of higher-order combinatorial matching not only with geodesic neighborhood, 


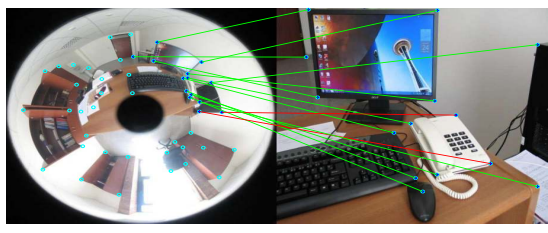

(a)

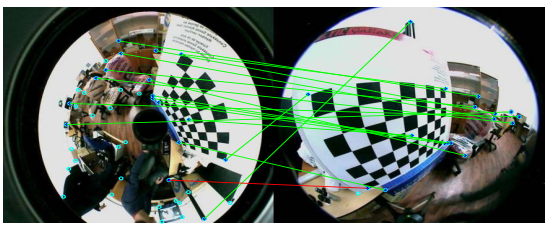

(b)

Fig. 4. Instances of matchings between (a) Desktop omnidirectional and planar images and (b) Chessboard omnidirectional and fish-eye images. Green/red lines show correct/incorrect matches, respectively. Isolated points show no matches.

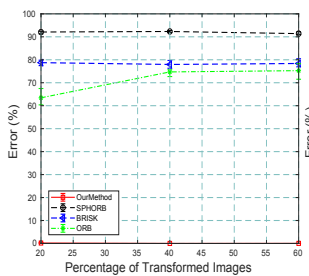

(a)

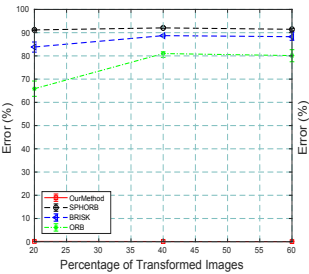

(b)

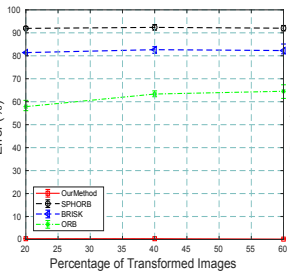

(c)

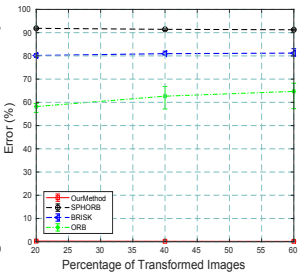

(d)

Fig. 5. Error $(\%)$ in matching when varying the percentage (20\% to 60\%) of transformed images in the set of spherical images of Desktop $(a)-(d)$. (a) $40^{\circ}$ rotation, (b) reflection, $(c)$ scaling and (d) shear.

but also with euclidean neighborhood, we run our planar variant (OurPlanar) on popular 2D image datasets, competing with standard matching algorithms. We pick four wellknown difficult matching datasets (Books, Building, Magazine, and Butterfly) that suffer from heavy occlusions and non-affine transformations [31]. Results for such an experiment are shown in Table 4 From the results, we observe that our method also serves as a powerful planar matching method and is still competitive using an euclidean neighborhood for our affine weight vectors. It significantly outperforms both the popular planar matching methods.

Table 4. Error $(\%)$ of pairwise matching between planar images of four datasets for different methods.

\begin{tabular}{lllll}
\hline Algorithms & Magazine & Building & Books & Butterfly \\
\hline OurPlanar & $\mathbf{0 . 0} \pm \mathbf{0 . 0} \%$ & $\mathbf{1 . 0 3} \pm \mathbf{0 . 0 1} \%$ & $\mathbf{1 9 . 7 2} \pm \mathbf{0 . 2 0} \%$ & $\mathbf{0 . 0} \pm \mathbf{0 . 0} \%$ \\
FGM & $\mathbf{0 . 0} \pm \mathbf{0 . 0} \%$ & $74.87 \pm 0.07 \%$ & $97.54 \pm 0.01 \%$ & $16.12 \pm 0.0 \%$ \\
Tensor & $\mathbf{0 . 0} \pm \mathbf{0 . 0} \%$ & $43.24 \pm 2.98 \%$ & $32.35 \pm 0.15 \%$ & $1.07 \pm 0.17 \%$ \\
\hline
\end{tabular}

RANSAC: Geometric Verification and Rectification We also performed fundamental matrix based geometric verification using RANSAC algorithm [16] after descriptor based matching on two datasets for spherical and their planar versions for SPHORB 
and ORB in Table 5 . We observed that the results are improved (but still not better than our proposed method) in some cases but prune a lot of matches. Nearly $40-50 \%$ of matches are considered as outliers which makes it difficult to handle the noise. On the other hand, our method performs much better in any case while considering outliers.

Table 5. Error (\%) of pairwise matching between spherical images of Desktop and Parking datasets and on their unwrapped versions for verification.

\begin{tabular}{lllll}
\hline Algorithms & Desktop & Desktop_flat & Parking & Parking_flat \\
\hline OurWarped & $\mathbf{0 . 3 2} \pm \mathbf{0 . 0} \%$ & $\mathbf{1 . 0 6} \pm \mathbf{0 . 0} \%$ & $\mathbf{0 . 0} \pm \mathbf{0 . 0} \%$ & $\mathbf{0 . 0} \pm \mathbf{0 . 0} \%$ \\
SPHORB+RANSAC & $96.1 \pm 0.0 \%$ & $93.9 \pm 0.0 \%$ & $95.0 \pm 0.0 \%$ & $100.0 \pm 0.0 \%$ \\
ORB+RANSAC & $29.3 \pm 0.0 \%$ & $70.6 \pm 0.0 \%$ & $55.0 \pm 0.0 \%$ & $100.0 \pm 0.0 \%$ \\
\hline
\end{tabular}

We performed rectification [5] on spherical images of Desktop dataset followed by BRISK descriptor for matching. The results improved from $78.9 \%$ (in Table 2 ) to $52.11 \%$ error. However, we observed that despite these improvements, our method still outperforms them. Also, in most of the cases, the rectification algorithm does not perform well and outputs noisy or distorted images. So, there is no guarantee to find the best solution.

Ablative Studies (Effect of Affine Transformation) We remove completely at random $40-80 \%$ of landmark points on the Desktop dataset, and introduce affine transformations on these points. Figure 5 shows the results of affine transformation like rotation, reflection, scaling, and shear. We rotated images (clockwise) by $40^{\circ}$ and performed matching for four algorithms. Then, we generated mirror images along the $x$-axis from the same dataset to introduce reflection. We also conducted transformation by scaling and shear of $360^{\circ}$ images. We resized images in both the directions with scales 0.5 and 1.5 randomly. For shearing, we stretched images with 0.5 factor along $y$-axis. For all types of transformations, we observe that the results shown in Figure 5 clearly indicates that our method is robust to all kinds of affine transformations and easily outperforms other state-of-the-art methods.

\section{Conclusion}

We presented a bijective assignment between sets of landmark points embedded on a pair of images warped onto curved manifolds by the following steps. First, we built a graph induced simplicial complex on the warped images. Second, we proposed a constrained QAP that matches corresponding co-dimensional simplices between two simplicial complexes along with an efficient algorithm to solve the constrained QAP. Finally, we conducted extensive experiments, broadly grouped as comparative matching and ablative studies, in order to gain insight into the accuracy and robustness of our method. We are currently exploring the possibility of integrating such high-dimensional combinatorial structures into Spherical CNNs [6] to capture higher-order and latent structure. 


\section{References}

1. Ahmed, A.I., Aldhaheri, S.R., Rodriguez Kovacs, J., Narasimhulu, D., Putra, M., Minkoff, H., Haberman, S.: Sonographic measurement of cervical volume in pregnant women at high risk of preterm birth using a geometric formula for a frustum versus 3-dimensional automated virtual organ computer-aided analysis. Journal of Ultrasound in Medicine 36(11), 2209-2217 (2017)

2. Alahi, A., Ortiz, R., Vandergheynst, P.: FREAK: Fast retina keypoint. In: CVPR. pp. 510-517. IEEE (2012)

3. Azevedo, R.G.d.A., Birkbeck, N., De Simone, F., Janatra, I., Adsumilli, B., Frossard, P.: Visual distortions in 360-degree videos. arXiv preprint arXiv:1901.01848 (2019)

4. Bay, H., Tuytelaars, T., Van Gool, L.: SURF: Speeded up robust features. In: ECCV. pp. 404-417. Springer (2006)

5. Bradski, G., Kaehler, A.: Learning OpenCV: Computer vision with the OpenCV library. O’Reilly Media Inc. (2008)

6. Cohen, T.S., Geiger, M., Köhler, J., Welling, M.: Spherical CNNs. ICLR (2018)

7. Colombo, C., Bimbo, A.D., Pernici, F.: Image mosaicing from uncalibrated views of a surface of revolution. In: Proc. BMVC. pp. 43.1-43.10 (2004)

8. Coors, B., Paul Condurache, A., Geiger, A.: Spherenet: Learning spherical representations for detection and classification in omnidirectional images. In: Proceedings of the European Conference on Computer Vision (ECCV). pp. 518-533 (2018)

9. Cruz-Mota, J., Bogdanova, I., Paquier, B., Bierlaire, M., Thiran, J.P.: Scale invariant feature transform on the sphere: Theory and applications. International Journal of Computer Vision 98(2), 217-241 (2012)

10. Cyganek, B.: An Introduction to 3D Computer Vision Techniques and Algorithms. John Wiley \&\#38; Sons, Inc. (2007)

11. Dey, T.K., Fan, F., Wang, Y.: Graph induced complex on point data. Comput. Geom. Theory Appl. 48(8), 575-588 (2015)

12. Duchenne, O., Bach, F., Kweon, I.S., Ponce, J.: A tensor-based algorithm for high-order graph matching. IEEE Transactions on Pattern Analysis and Machine Intelligence (PAMI) 33(12), 2383-2395 (2011)

13. Feizi, S., Quon, G., Recamonde-Mendoza, M., Médard, M., Kellis, M., Jadbabaie, A.: Spectral alignment of networks. arXiv preprint arXiv:1602.04181 (2016)

14. Goesele, M., Snavely, N., Curless, B., Hoppe, H., Seitz, S.M.: Multi-view stereo for community photo collections. In: ICCV. pp. 1-8. IEEE (2007)

15. Guan, H., Smith, W.A.: BRISKS: Binary features for spherical images on a geodesic grid

16. Hartley, R., Zisserman, A.: Multiple view geometry in computer vision. Cambridge university press (2003)

17. Kalesnykiene, V., Kamarainen, J.k., Lensu, L., Sorri, I., Uusitalo, H., Kälviäinen, H., Pietilä, J.: Diaretdb0: evaluation database and methodology for diabetic retinopathy algorithms

18. Kaminsky, R.S., Snavely, N., Seitz, S.M., Szeliski, R.: Alignment of 3d point clouds to overhead images. In: 2009 IEEE Computer Society Conference on Computer Vision and Pattern Recognition Workshops. pp. 63-70. IEEE (2009)

19. Leordeanu, M., Zanfir, A., Sminchisescu, C.: Semi-supervised Learning and Optimization for Hypergraph Matching. In: IEEE International Conference on Computer Vision (ICCV) (November 2011)

20. Leordeanu, M., Hebert, M.: A spectral technique for correspondence problems using pairwise constraints. In: ICCV. vol. 2, pp. 1482-1489. IEEE (2005)

21. Leutenegger, S., Chli, M., Siegwart, R.Y.: BRISK: Binary robust invariant scalable keypoints. In: ICCV. pp. 2548-2555. IEEE (2011) 
22. Li, H., Huang, X., He, L.: Object matching using a locally affine invariant and linear programming techniques. IEEE Transactions on Pattern Analysis \& Machine Intelligence (2), 411-424 (2013)

23. Li, Y., Gregori, G., Lam, B.L., Rosenfeld, P.J.: Automatic montage of sd-oct data sets. Opt. Express 19(27), 26239-26248 (2011)

24. Lowe, D.G.: Distinctive image features from scale-invariant keypoints. International Journal of Computer Vision 60(2), 91-110 (2004)

25. Munkres, J.R.: Elements of algebraic topology. Addison-Wesley (1984)

26. Olivier Pauly, Gozde Unal, G.S.S.C.T.F.: Semi-automatic matching of oct and ivus images for image fusion (2008), https://doi.org/10.1117/12.773805

27. Rosten, E., Drummond, T.: Machine learning for high-speed corner detection. In: ECCV. pp. 430-443. Springer (2006)

28. Rublee, E., Rabaud, V., Konolige, K., Bradski, G.: ORB: An efficient alternative to SIFT or SURF. In: ICCV. pp. 2564-2571. IEEE (2011)

29. Scaramuzza, D., Martinelli, A., Siegwart, R.: A toolbox for easily calibrating omnidirectional cameras. In: 2006 IEEE/RSJ International Conference on Intelligent Robots and Systems. pp. 5695-5701. IEEE (2006)

30. Sengupta, S., Singh, A., Leopold, H.A., Lakshminarayanan, V.: Ophthalmic diagnosis and deep learning - a survey (2018)

31. Sharma, C., Nathani, D., Kaul, M.: Solving partial assignment problems using random clique complexes. In: ICML. pp. 4593-4602 (2018)

32. Shen, D., Davatzikos, C.: HAMMER: Hierarchical attribute matching mechanism for elastic registration. IEEE transactions on medical imaging 21(11), 1421-1439 (2002)

33. Starck, J., Hilton, A.: Spherical matching for temporal correspondence of non-rigid surfaces. In: Tenth IEEE International Conference on Computer Vision (ICCV'05) Volume 1. vol. 2, pp. 1387-1394. IEEE (2005)

34. Sung, B., Kim, S.H., Lee, S., Lim, J., Lee, J.K., Soh, K.S.: Nanofluid transport in a living soft microtube. Journal of Physics D: Applied Physics (2015)

35. Szeliski, R.: Computer vision: algorithms and applications. Springer Science \& Business Media (2010)

36. Yang, J., Li, H., Jia, Y.: Go-icp: Solving 3d registration efficiently and globally optimally. In: Proceedings of the IEEE International Conference on Computer Vision. pp. 1457-1464 (2013)

37. Zeng, A., Song, S., Nießner, M., Fisher, M., Xiao, J., Funkhouser, T.: 3dmatch: Learning local geometric descriptors from rgb-d reconstructions. In: Proceedings of the IEEE Conference on Computer Vision and Pattern Recognition. pp. 1802-1811 (2017)

38. Zhao, Q., Feng, W., Wan, L., Zhang, J.: SPHORB: A fast and robust binary feature on the sphere. International Journal of Computer Vision 113(2), 143-159 (2015)

39. Zhou, F., De la Torre, F.: Factorized Graph Matching. IEEE Transactions on Pattern Analysis and Machine Intelligence (PAMI) 38(9), 1774-1789 (2016)

40. Zhou, F., De la Torre, F.: Deformable graph matching. In: CVPR. pp. 2922-2929 (2013) 


\section{A Proof of Lemma 1}

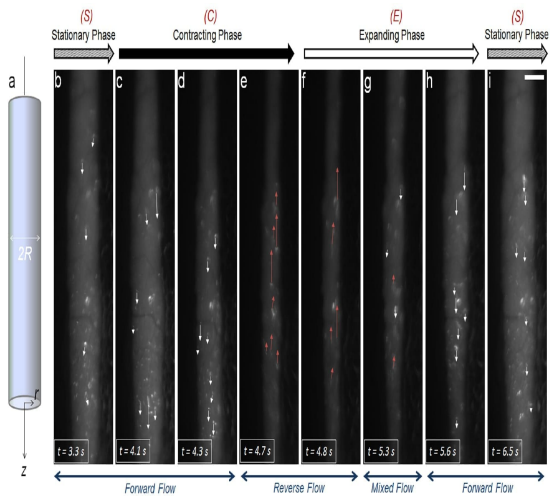

(a)

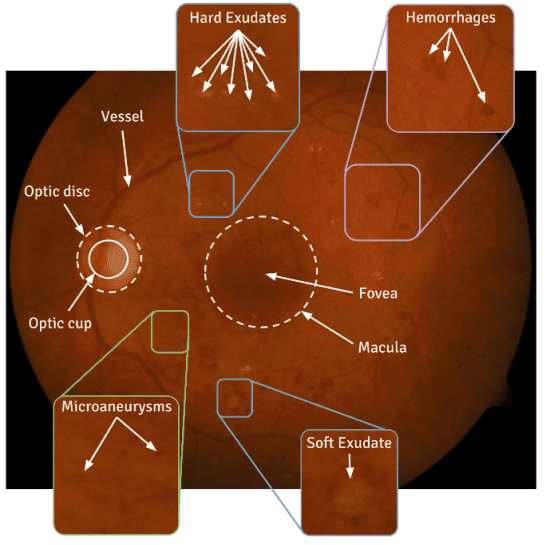

(b)

Fig. 6. Example of (a) a nanofluid microflow pattern monitored by tracking the colloidal microparticles [34], (b) a fundus image with retinal morphologies and pathologies [?].

We begin this proof by focusing on the case where $k \geq 2$. We know that each $k$-clique contains $\left(\begin{array}{l}k \\ 2\end{array}\right)$ edges in it.

Now, we study how many $k$-cliques an arbitrary edge $(u, v)$ can belong to. Notice that if edge $(u, v)$ belongs to a $k$-clique $K$, then all other vertices in the $K$ must also be adjacent to $u$ and vertex $u$ can have at most $\delta-1$ neighbors that are not $v$. Therefore, if $K$ contains both vertices $u$ and $v$, then $K$ contains $k-2$ other vertices and each of them must also be neighbors of $u$. So, combining all these observations, if a $k$-clique $K$ must contain both $u$ and $v$ and $k-2$ of the other $\delta-1$ maximum allowed neighbors of $u$, then there must be at most $\left(\begin{array}{l}\delta-1 \\ k-2\end{array}\right)$ such $k$-cliques.

Thus, $G$ has at most $\frac{m\left(\begin{array}{c}\delta-1 \\ k-2\end{array}\right)}{\left(\begin{array}{l}k \\ 2\end{array}\right)} k$-cliques. Then, the total number of $k$-cliques in $G$ for $k \geq 2$ is

$$
\begin{aligned}
\sum_{k=2}^{h} \frac{m\left(\begin{array}{l}
\delta-1 \\
k-2
\end{array}\right)}{\left(\begin{array}{l}
k \\
2
\end{array}\right)} & =m \sum_{k=2}^{h} \frac{2}{k(k-1)} \frac{(\delta-1) !}{(k-2) !(\delta+1-k) !} \\
& =m \sum_{k=2}^{h} \frac{2(\delta-1) !}{k !(\delta+1-k) !}
\end{aligned}
$$


We need a $(\delta+1)$ ! term in the numerator of Equation QAP, since we know that $h \leq$ $(\delta+1)$. So,

$$
\begin{aligned}
& m \sum_{k=2}^{h} 2\left\{\frac{(\delta+1) !}{(\delta+1)(\delta)}\right\} \frac{1}{k !(\delta+1-k) !} \\
& =\frac{2 m}{\delta(\delta+1)} \sum_{k=2}^{h} \frac{(\delta+1) !}{k !(\delta+1-k) !} \\
& =\frac{2 m}{\delta(\delta+1)}\left(\sum_{k=0}^{h} \frac{(\delta+1) !}{k !(\delta+1-k) !}-\frac{(\delta+1) !}{1 ! \delta !}-\frac{(\delta+1) !}{0 !(\delta+1) !}\right) \\
& =\frac{2 m}{\delta(\delta+1)}\left(\left\{\sum_{k=0}^{h}\left(\begin{array}{c}
\delta+1 \\
k
\end{array}\right)\right\}-\delta-2\right) \\
& \leq \frac{2 m}{\delta(\delta+1)}\left[\min \left\{(\delta+1)^{h}+1,\left(\frac{e(\delta+1)}{h}\right)^{h}\right\}-\delta-2\right]
\end{aligned}
$$

In Equation 8 , the term in braces \{\} , is the partial sum of the first $h$ binomial coefficients. Note that for $h=\delta+1$, the term would reduce to $2^{\delta+1}$. Finally, we must also account for the $n 1$-cliques (vertices) in $G$. This completes the proof.

\section{B Additional Experiments}

\section{B.1 Datasets}

Details of all six datasets used for warped image matching are as follows: (i) Chessboard dataset has 28 spherical images in which 16 are omnidirectional and 12 are fish-eye images. The images contain a view of a room with a chessboard, chair, table, people, and computer systems. (ii) Kamaishi dataset has 15 panoramic images of video frames from a moving car. (iii) We picked 10 panoramic images covering various scenes from SUN360 dataset. (iv) Desktop dataset consists of two kinds of images (7 omnidirectional and 9 planar). Images represent a room in which a desktop is positioned on a table. $(v)$ Parking dataset also has omnidirectional and planar images with a view outside a house near a parking area. (vi) Table dataset contains omnidirectional, fish-eye, and planar images. This dataset has few tables in the images with computer systems, boards, chairs, etc. placed in a room. All our annotated datasets will be made publicly available.

In addition to experiments in our main paper, we conducted additional experiments for different settings. In multi-modal matching, we performed matching on unwrappedunwrapped spherical/warped images and unwrapped spherical/warped vs planar images. In ablative studies, we perfomed experiments considering two noise models and geometric verification and rectification. Additional experiments on rotation are performed for SUN360 dataset and Desktop dataset. We also analyzed the effect of missing points on four datasets (Chessboard, Desktop, Kamaishi and Table). Additionally, we also studied effects of varying $k$ values in a neighborhood of landmark points, varying the radius of the sphere and manifold, and varying the number of landmark points for matching. Matching 


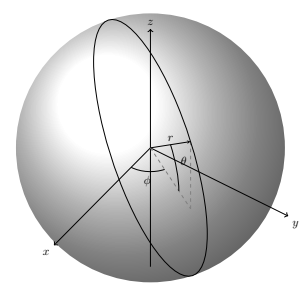

Fig. 7. Parameterized $\mathcal{S}^{2}$ (for Example 2 in main draft)

images for different cases are shown in Figures 16 and 17 . We also created a video of frames of Kamaishi dataset where we match points of frames in a sequence which can be useful for tracking purposes. The video titled Kamaishi_Panorama_Matching_Video.avi is located in the folder of our supplementary material.

Table 6. Error (\%) of pairwise matching between spherical (omnidirectional and fish-eye) and planar images of three datasets for different methods.

\begin{tabular}{llll}
\hline Algorithms & Desktop & Parking & Table \\
\hline OurWarped_Cone & $14.71 \pm 0.0 \%$ & $4.41 \pm 0.0 \%$ & $0.88 \pm 0.0 \%$ \\
OurWarped_Ellip & $13.82 \pm 0.0 \%$ & $5.94 \pm 0.0 \%$ & $2.65 \pm 0.0 \%$ \\
\hline OurSpherical & $\mathbf{1 5 . 1 6} \pm \mathbf{0 . 0} \%$ & $\mathbf{5 . 5 3} \pm \mathbf{0 . 0} \%$ & $\mathbf{0 . 7 8} \pm \mathbf{0 . 0} \%$ \\
FGM + geodesic & $97.94 \pm 0.24 \%$ & $97.53 \pm 0.57 \%$ & $96.98 \pm 2.51 \%$ \\
SPHORB & $41.5 \pm 0.0 \%$ & $53.85 \pm 0.0 \%$ & $70.88 \pm 0.0 \%$ \\
BRISK & $37.84 \pm 0.0 \%$ & $51.05 \pm 0.0 \%$ & $65.11 \pm 0.0 \%$ \\
ORB & $34.82 \pm 0.0 \%$ & $48.82 \pm 0.0 \%$ & $62.78 \pm 0.0 \%$ \\
Tensor & $93.85 \pm 0.0 \%$ & $94.43 \pm 0.22 \%$ & $56.94 \pm 1.02 \%$ \\
FGM & $98.0 \pm 0.0 \%$ & $97.50 \pm 0.01 \%$ & $97.5 \pm 0.01 \%$ \\
\hline
\end{tabular}

\section{B.2 Matching on Spherical/Warped vs. Planar}

To understand the effects of matching spherical / warped images versus planar images, we conducted matching experiments between planar and warped images, whose results are shown in the first two rows of Table 6. We find that the matching error is higher for warped-planar matching compared to warped-warped matching shown in Table in main paper because of the difference in geodesic distances between landmarks embedded on the warped versus the Euclidean distance between landmarks on planar images.

Next, planar images are compared to both fish-eye and omnidirectional images. The results are shown in Table 6 in which we clearly observe an increase in error for the Desktop dataset when compared to the omni-omni matching results in Table in main paper. As planar images cover a much smaller portion of the scenes as compared to omnidirectional images, they also contain much fewer landmark points with many 
occluded regions, which in turn affects our method due to the lack of higher-dimensional cliques. However, our method still beats other state-of-the-art methods.

\section{B.3 Matching on Planar vs. Unwrapped Spherical/Warped}

We observe that matching between spherical and planar is a challenge due to the difference in euclidean and geodesic distances in planar and spherical images, respectively, as is evidenced in Table 3 of our main paper. Therefore, to compare these kinds of images, we can perform matching between unwrapped equirectangular spherical/warped and planar images. In this way, we can also apply state-of-the-art graph matching methods which perform well on planar images. Thus, we consider three datasets with both spherical (omnidirectional and fish-eye) and planar images and conducted the experiment. Table 7 shows the results. We can compare the results with Table 3 in the main paper, we can clearly see that the results are almost same even after flattening of the image. Thus, comparing flattened and planar images does not reduce the error of matchings between spherical and planar images. However, our method outperforms the existing methods.

Table 7. Error (\%) of pairwise matching between unwrapped equirectangular version of spherical (omnidirectional and fish-eye) images and planar images of three datasets for different methods including graph matching methods on flat surfaces.

\begin{tabular}{llll}
\hline Algorithms & Desktop & Parking & Table \\
\hline \hline OurWarped_Cone & $14.26 \pm 0.0 \%$ & $5.23 \pm 0.0 \%$ & $1.09 \pm 0.0 \%$ \\
OurWarped_Ellip & $15.16 \pm 0.0 \%$ & $3.59 \pm 0.0 \%$ & $2.96 \pm 0.0 \%$ \\
\hline OurSpherical & $\mathbf{1 3 . 0 8} \pm \mathbf{0 . 0} \%$ & $\mathbf{4 . 1 0} \pm \mathbf{0 . 0} \%$ & $\mathbf{0 . 9 3} \pm \mathbf{0 . 0} \%$ \\
RCC & $30.85 \pm 1.78 \%$ & $12.38 \pm 1.53 \%$ & $11.69 \pm 1.58 \%$ \\
EigenAlign & $99.94 \pm 0.0 \%$ & $99.22 \pm 0.0 \%$ & $99.25 \pm 0.0 \%$ \\
Tensor & $97.88 \pm 0.11 \%$ & $95.59 \pm 0.35 \%$ & $81.04 \pm 1.43 \%$ \\
FGM & $99.0 \pm 0.0 \%$ & $100.0 \pm 0.0 \%$ & $97.0 \pm 0.0 \%$ \\
SPHORB & $41.33 \pm 0.0 \%$ & $59.9 \pm 0.0 \%$ & $71.15 \pm 0.0 \%$ \\
BRISK & $37.67 \pm 0.0 \%$ & $55.98 \pm 0.0 \%$ & $67.8 \pm 0.0 \%$ \\
ORB & $34.43 \pm 0.0 \%$ & $45.03 \pm 0.0 \%$ & $64.84 \pm 0.0 \%$ \\
\hline
\end{tabular}

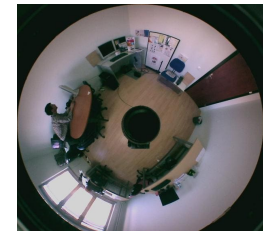

(a)

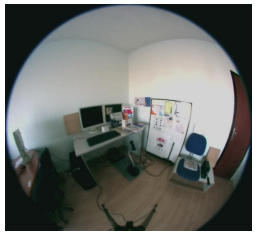

(b)

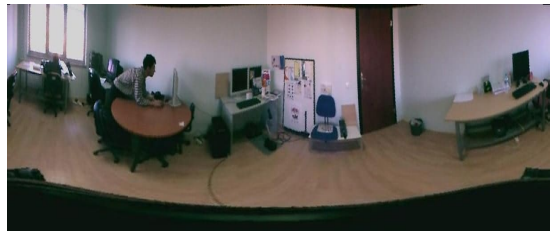

(c)

Fig. 8. Example of (a) a parabolic omnidirectional image, (b) fish-eye, and (c) panoramic (unwrapped equirectangular) images of the same view. 


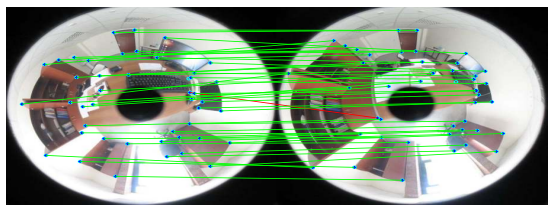

(a)

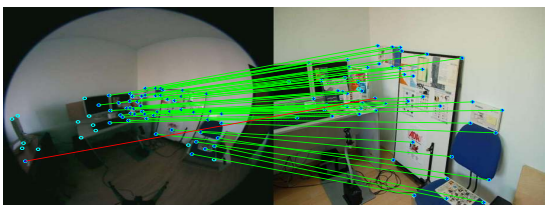

(b)

Fig. 9. Instances of matchings between (a) Desktop omnidirectional images and (b) Table fish-eye and planar images. Green/red lines show correct/incorrect matches respectively. Isolated points show no matches.

\section{B.4 Effect of Noise Models}

Our proposed matching method is subjected to two random noise models proposed by Feizi et. al. [13]. While matching two simplicial complexes $\mathcal{M}$ and $\mathcal{M}^{\prime}$, noise is introduced in the first simplicial complex $\mathcal{M}$. We denote the noisy simplicial complex as $\widetilde{\mathcal{M}}$. Next, we attempt a matching between $\widetilde{\mathcal{M}}$ and $\mathcal{M}^{\prime}$. The noisy complex $\widetilde{\mathcal{M}}$ is generated as follows:

Noise Model I: $\widetilde{\mathcal{M}}=\mathcal{M} \odot(1-P)+(1-\mathcal{M}) \odot P$

Noise Model II: $\widetilde{\mathcal{M}}=\mathcal{M} \odot(1-P)+(1-\mathcal{M}) \odot Q$

where, $\odot$ represents the element-wise multiplication with matrices $P / Q$ which are

Table 8. Error (\%) of pairwise matching between unwrapped spherical (omnidirectional and fish-eye) images of four datasets for different graph matching methods with two noise models.

\begin{tabular}{lllll}
\hline Algorithms & Chessboard & Desktop & Parking & Table \\
\hline \hline \multicolumn{5}{c}{ Noise Model I } \\
\hline OurMethod & $\mathbf{4 . 1 7} \pm \mathbf{0 . 0} \%$ & $\mathbf{0 . 8 5} \pm \mathbf{0 . 0} \%$ & $\mathbf{0 . 0} \pm \mathbf{0 . 0} \%$ & $\mathbf{0 . 3 1} \pm \mathbf{0 . 0} \%$ \\
RCC & $29.7 \pm 0.68 \%$ & $17.1 \pm 1.17 \%$ & $15.8 \pm 7.89 \%$ & $16.2 \pm 0.48 \%$ \\
EigenAlign & $99.5 \pm 0.0 \%$ & $96.5 \pm 0.0 \%$ & $97.7 \pm 0.0 \%$ & $98.2 \pm 0.0 \%$ \\
FGM & $93.0 \pm 0.0 \%$ & $72.0 \pm 0.0 \%$ & $65.0 \pm 0.0 \%$ & $92.0 \pm 0.0 \%$ \\
\hline \multicolumn{5}{c}{ Noise Model II } \\
\hline OurMethod & $\mathbf{4 . 3 6} \pm \mathbf{0 . 0} \%$ & $\mathbf{0 . 6 4} \pm \mathbf{0 . 0} \%$ & $\mathbf{0 . 0} \pm \mathbf{0 . 0} \%$ & $\mathbf{0 . 4 4} \pm \mathbf{0 . 0} \%$ \\
RCC & $29.9 \pm 0.52 \%$ & $17.0 \pm 1.01 \%$ & $14.2 \pm 5.26 \%$ & $16.1 \pm 0.42 \%$ \\
EigenAlign & $98.9 \pm 0.0 \%$ & $97.2 \pm 0.0 \%$ & $98.5 \pm 0.0 \%$ & $99.1 \pm 0.0 \%$ \\
FGM & $94.0 \pm 0.0 \%$ & $71.0 \pm 0.0 \%$ & $60.0 \pm 0.0 \%$ & $92.5 \pm 0.0 \%$ \\
\hline
\end{tabular}

binary random symmetric matrices drawn from a Bernoulli distribution with $v$ vertices and $p / q$ probabilities, respectively. Here, $P$ flips the vertex-vertex adjacency with probability $p$ and $Q$ adds new edges between non-connected vertices with probability $q$ to the underlying geometric graph $G=(V, E, g)$ of the simplicial complex $\mathcal{M}$. Experiments are performed between pairwise flattened (unwrapped) spherical images of four datasets against graph matching methods. Table 8 shows matching results after the application of both noise models and it shows that our method is the most robust. 


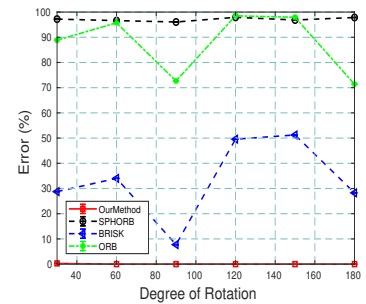

(a)

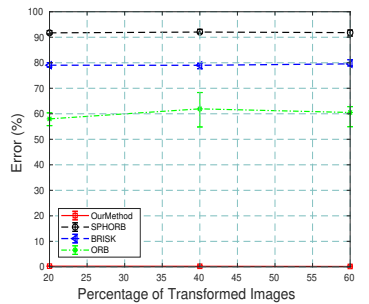

(b)

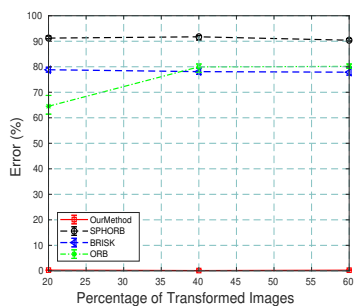

(c)

Fig. 10. Error(\%) of matching $(a)$ on SUN 360 dataset. We selected 10 spherical images and created their rotated versions. Matching pairs of images for different angles from $30^{\circ}$ to $180^{\circ}$ rotation. $(b)-(c)$ when varying the percentage ( $20 \%$ to $60 \%$ ) of transformed images in the set of spherical images of Desktop for $20^{\circ}$ and $60^{\circ}$ rotation respectively.

\section{B.5 Effect of Rotation}

We study the effect of rotation (affine transformation) on a simulated dataset. We collected 10 different images from SUN360 dataset and annotated them. Therefore, the number of landmark points vary in all the images. The images are panoramic spherical images. We perform rotation on the images from $30^{\circ}$ to $180^{\circ}$ and compare the original image against the rotated images. Figure 11(b) to 11(d) are the rotated versions of the original image in Figure 11(a). Matching results are presented in Figure 10(a) against three feature descriptor methods. We note that, there is an increase in the error percentage for other methods as the degree of rotation increases from $30^{\circ}$ to $180^{\circ}$, except in the $90^{\circ}$ rotation case, as $90^{\circ}$ rotation does not introduce distortions in the image and the pairwise-distances between points can be preserved. Whereas, our method is quite stable to transformation by rotation. We also rotated images (clockwise) by $20^{\circ}$ and $60^{\circ}$ for omnidirectional images of Desktop dataset and randomly transformed $20 \%$ to $60 \%$ of images in a set of spherical images. Results for both the rotations are shown in Figures 10(b) and 10(c)

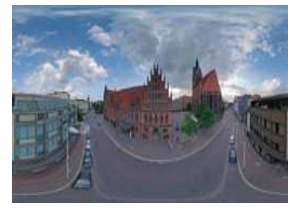

(a)

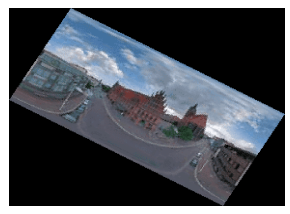

(b)

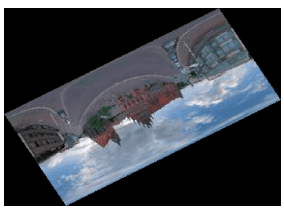

(c)

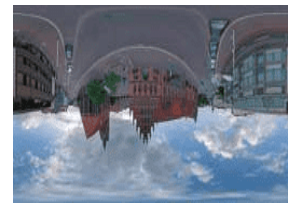

(d)

Fig. 11. (a) Original image from SUN 360 dataset, rotated images after $(b) 30^{\circ}$ rotation, $(c) 150^{\circ}$ rotation, and $(d) 180^{\circ}$ rotation. Matching results are shown in Figure 10(a) with the original image (a). 


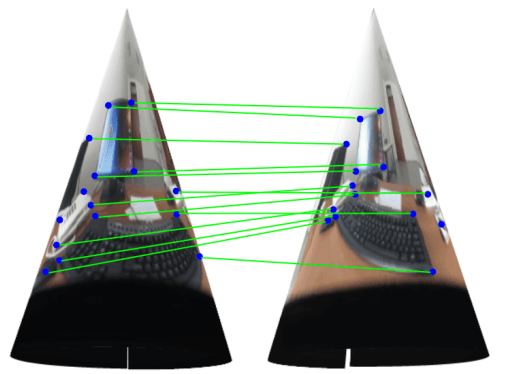

(a)

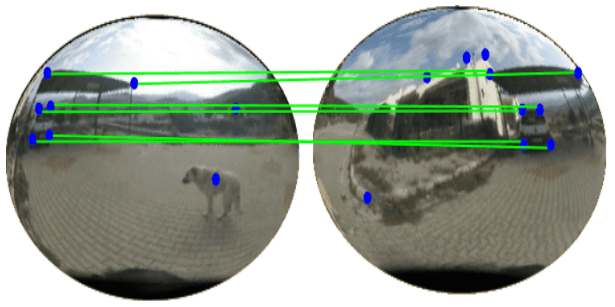

(b)

Fig. 12. Matching on warped images of (left) Cone and (right) Ellipsoid. Green lines show correct matches and isolated points show no matches.

\section{B.6 Effect of varying $k$-NN, Radius of surface and Number of points}

We study the effect of parameters $(k, R)$ and the effect of sparse / dense set of landmark points by performing few experiments for our method (spherical) and our warped on cone and ellipsoid methods and the existing feature descriptor based methods, respectively.

Vary $k$-Nearest Neighbour: We performed few experiments of matching between spherical vs. spherical (for Kamaishi dataset), spherical vs. planar (Desktop), unwrapped spherical vs. unwrapped spherical (Desktop) and unwrapped spherical vs. planar (Parking) images by varying $k$ parameter of $k$-NN while generating a simplicial complex. Figure 13(b) shows the results for four different datasets with $k$ varying from range 4 to 7 on $x$-axis. Although, the value of $k$ changes the matching results, the error doesn't change significantly. Selection of $k$ is important since it defines the local connectivity of a vertex. So, larger the value of $k$, larger are the adjacent vertices of a vertex in question. However, a large value of $k$ can slow down the algorithm and hence this trade-off must be taken into consideration while choosing $k$.

Vary Radius of Surface: Projecting an image onto a sphere or manifold depends on the radius of the underlying manifold. Change in radius affects the rate of distortion of projection onto the manifold. Thus, we conducted an experiment on spherical (omnidirectional) images of Desktop dataset. Results for varying $R$ ranging from 1 to 10 are shown in Figure 13(c) Matching is performed on our three methods including spherical (radius of 2-sphere), cone (radius of cone) and ellipsoid (radius of 2-sphere projected to an ellipse with a fixed major and minor axis). Results report that the change in radius doesn't affect the matching accuracy.

Sparse to Dense set of landmark points: We considered spherical (omnidirectional and fish-eye) images of Table dataset which consists of 93 landmark points originally. We randomly selected 10 to 90 landmark points on $x$-axis in Figure 13(a). This experiment analyzes if the matching algorithm depends on sparsity / density of landmark points in an image. From the results, our method doesn't seem to be affected by removal or addition of points whereas there is a substantial increase in error for other methods. 


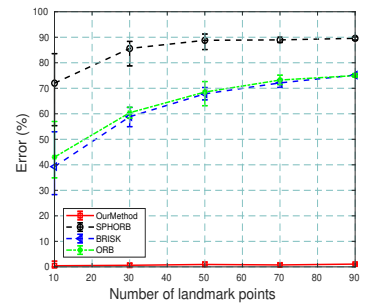

(a)

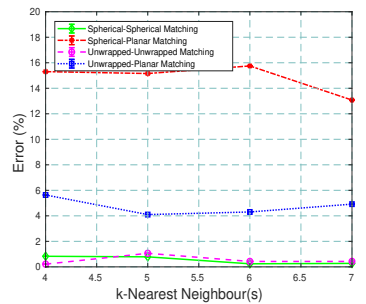

(b)

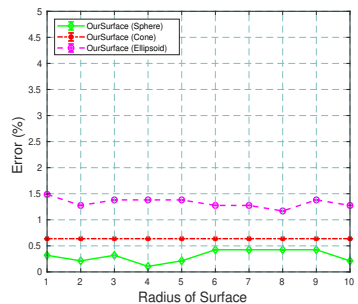

(c)

Fig. 13. Error(\%) of matching by $(a)$ varying the number of landmark points in the images of Table dataset(Sparse to Dense), (b) varying the value of $k$ (from $k=4$ to 7 ) in $k$-NN ball of a landmark point, and $(c)$ varying the radius of surface (from $r=1$ to 10) of a sphere, cone and an ellipsoid for Desktop dataset.

\section{B.7 Effect of Missing Points}

In order to compare the robustness of our method versus the state-of-the-art methods under the effect of landmark points missing completely at random (MCAR), we randomly remove $20 \%$ to $60 \%$ of the landmark points from $20 \%$ to $60 \%$ of images in a set of spherical images. We picked an arbitrary image as the original image and match it against the rest of the spherical images, affected by missing points, in the set. We conducted this experiment on four datasets mentioned in Figure 14 We have shown results for removal of points from $20 \%, 40 \%$ and $60 \%$ of images in Figure 14 . We observe that there is no large increase in error when $20 \%$ to $60 \%$ of the landmark points are removed randomly for our method. Also, results for other methods are not affected by varying percentage of missing points and there is no increase in the error percentage since removal of points reduces the number of points in matching, which in turn also reduces error for feature descriptor methods because of decrease in probability to mismatch. 


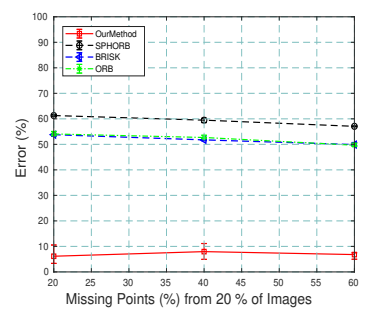

(a)

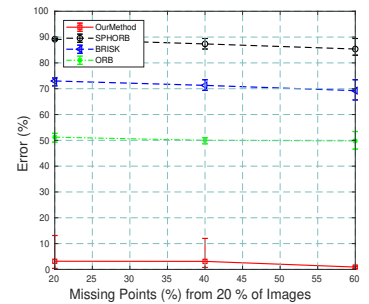

(d)

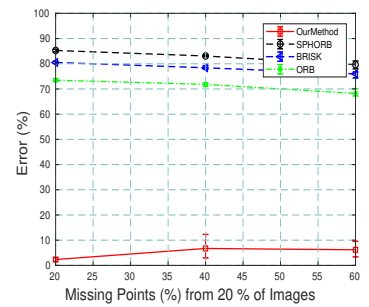

(g)

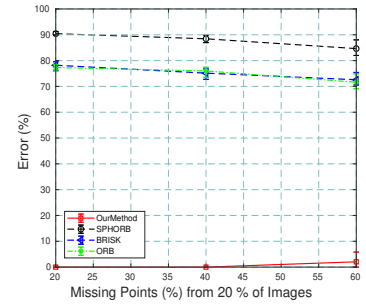

(j)

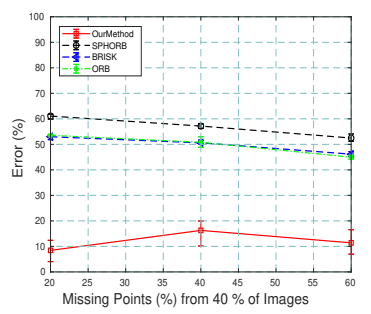

(b)

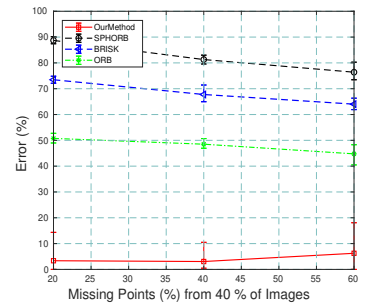

(e)

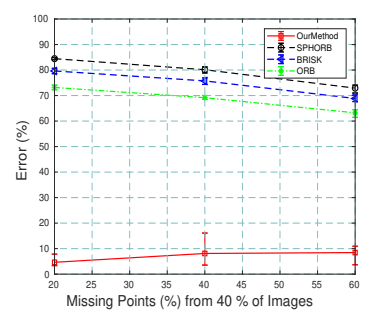

(h)

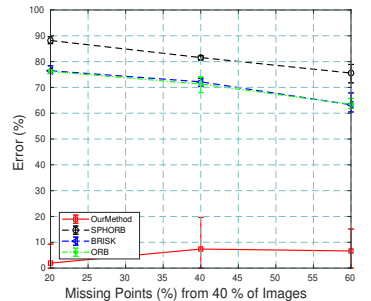

(k)

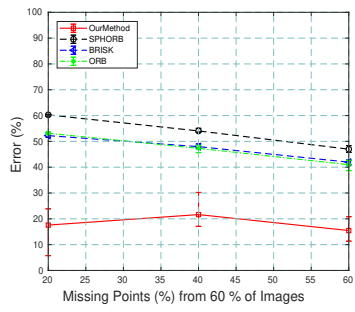

(c)

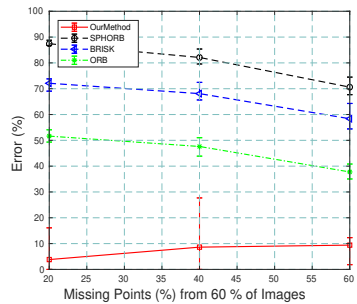

(f)

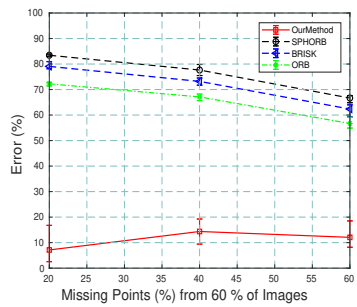

(i)

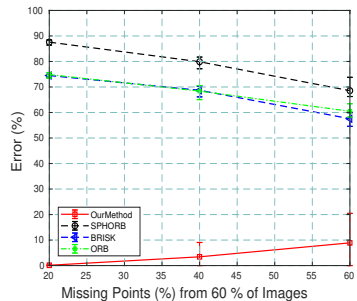

(1)

Fig. 14. Error (\%) of matching between spherical images by randomly removing points $(20 \%-$ $60 \%$ ) from $20 \%, 40 \%$ and $60 \%$ of the images. Matching is computed for four datasets $((a)-(c)$ Chessboard, $(d)-(f)$ Desktop, $(g)-(i)$ Kamaishi, $(j)-(l)$ Table) from $1^{\text {st }}$ frame to the other $N-1$ frames. 


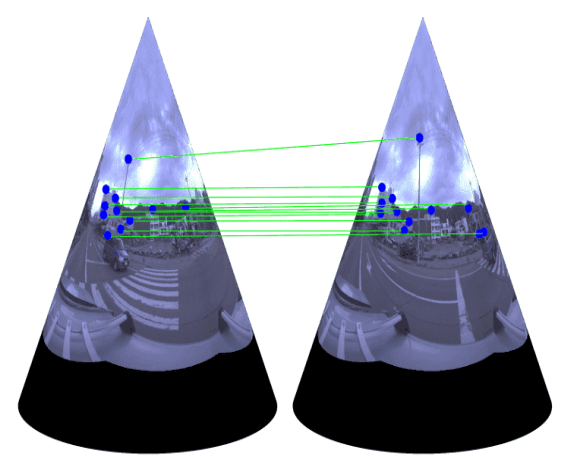

(a)

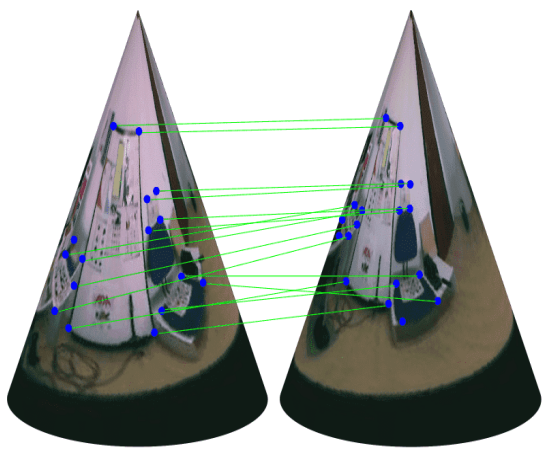

(c)

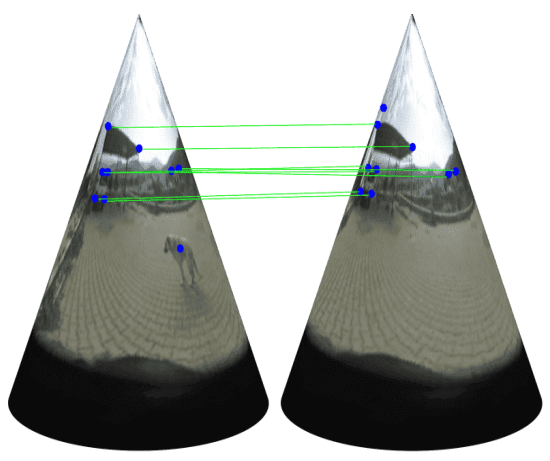

(e)

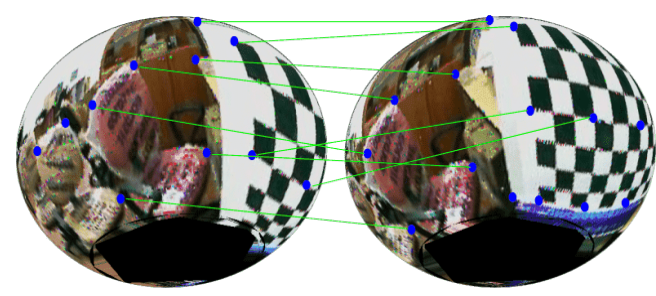

(b)

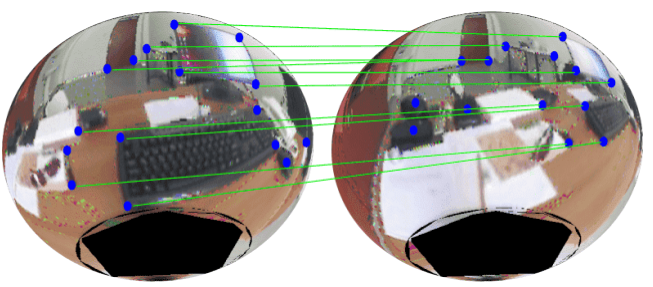

(d)

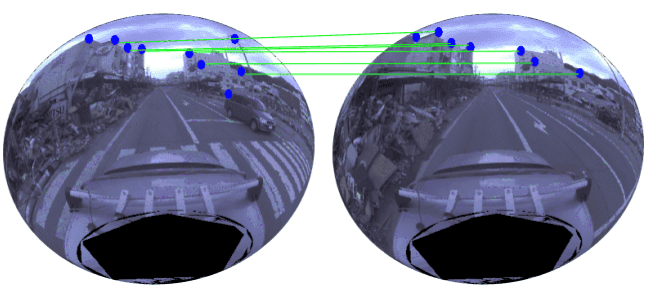

(f)

Fig. 15. Instances of a matching of unwrapped images of different datasets on curved manifold for $(a)$ Kamaishi, $(b)$ Chessboard, $(c)$ Table, $(d)$ Desktop, $(e)$ Parking and $(f)$ Kamaishi. Green lines show correct matches respectively. Isolated points show no matches. 


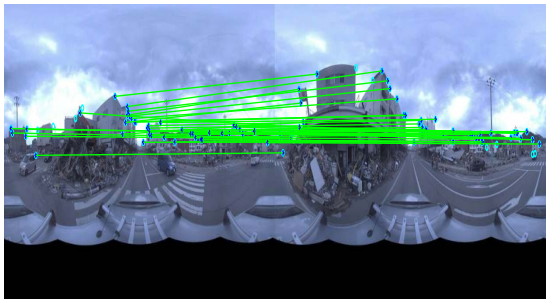

(a)

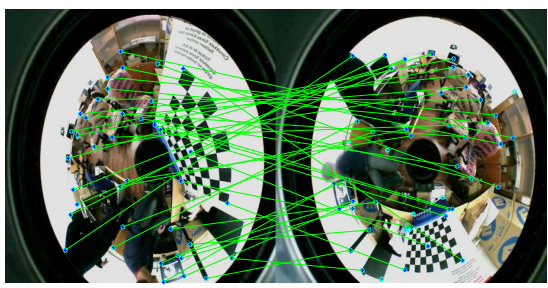

(c)

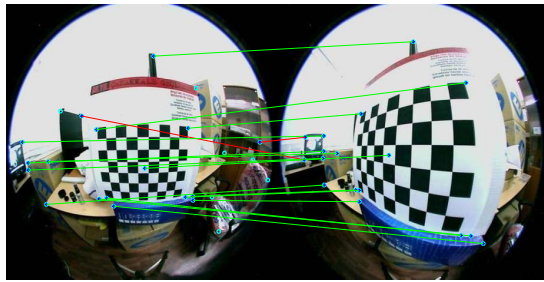

(e)

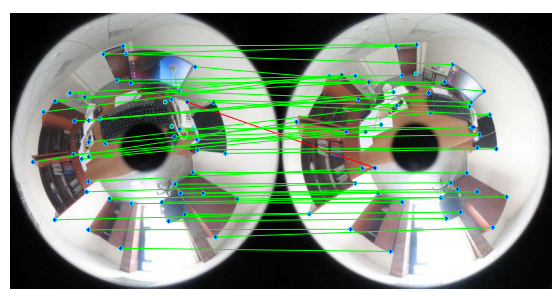

(g)

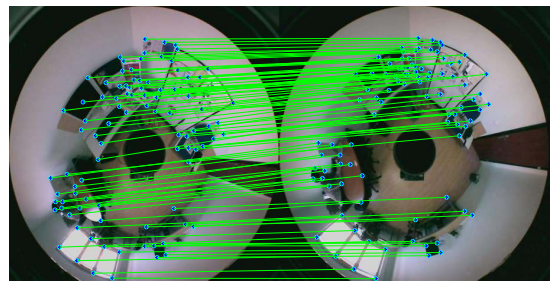

(i)

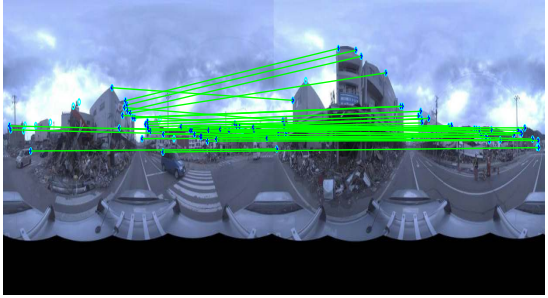

(b)

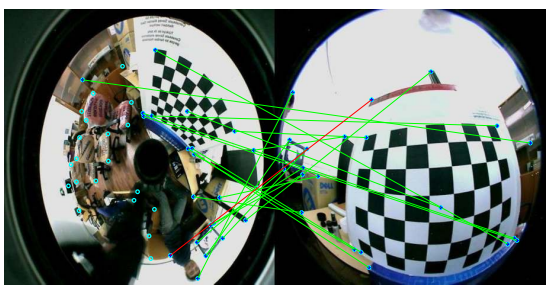

(d)

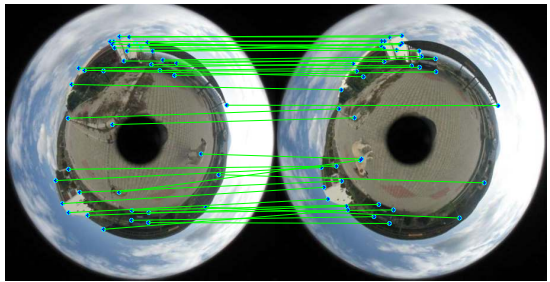

(f)

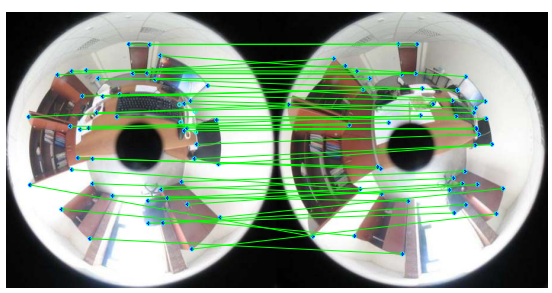

(h)

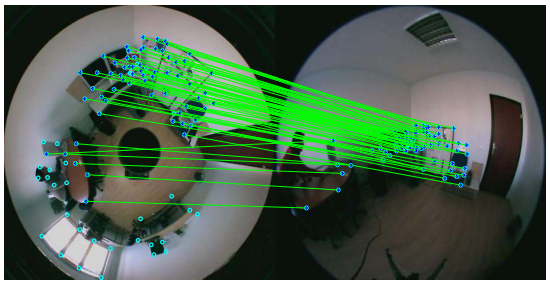

(j)

Fig. 16. Instances of matchings between spherical images of $(a)-(b)$ Kamaishi, $(c)-(e)$ Chessboard, $(f)$ Parking, $(g)-(h)$ Desktop and $(i)-(j)$ Table datasets. Green/red lines show correct/incorrect matches respectively. Isolated points show no matches. 


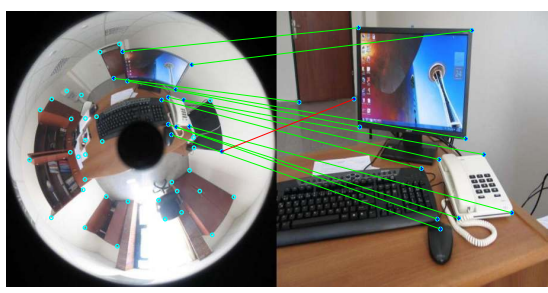

(a)

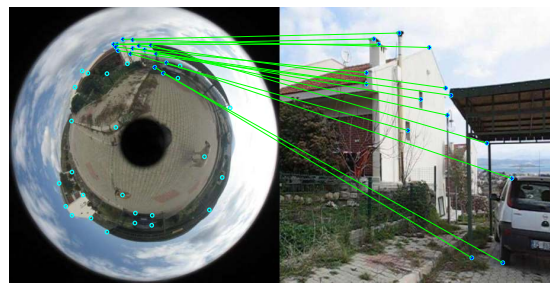

(c)

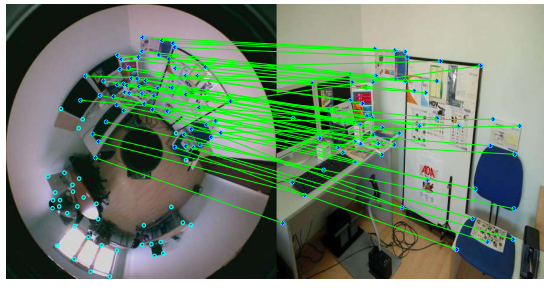

(e)

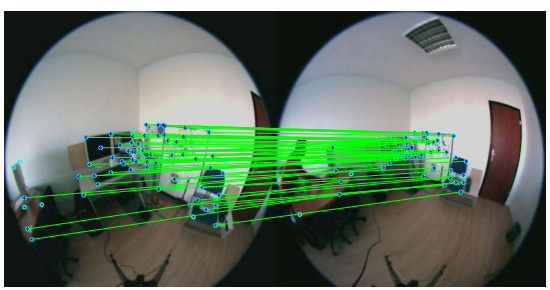

(g)

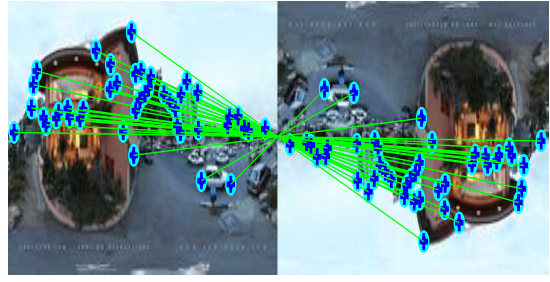

(i)

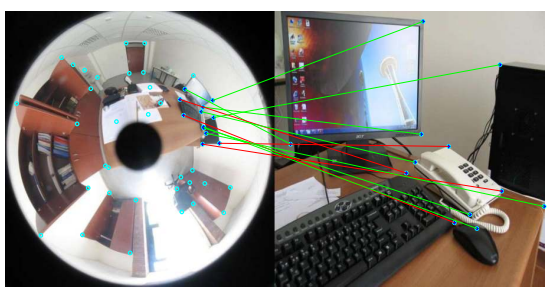

(b)

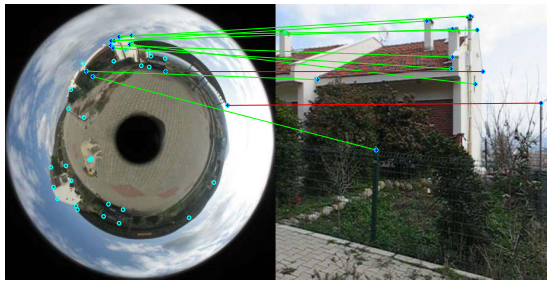

(d)

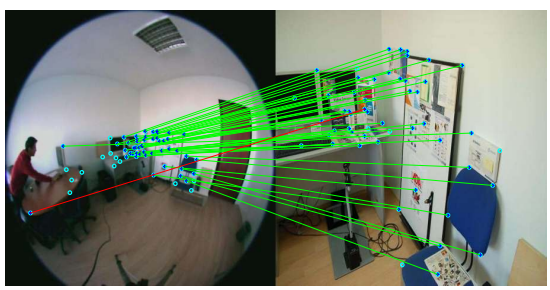

(f)

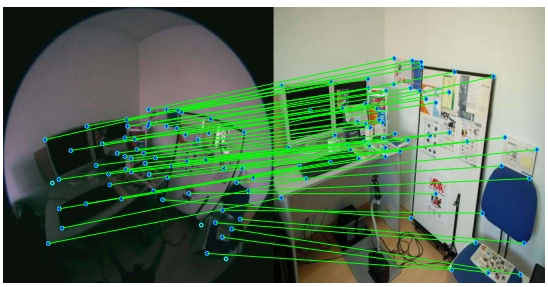

(h)

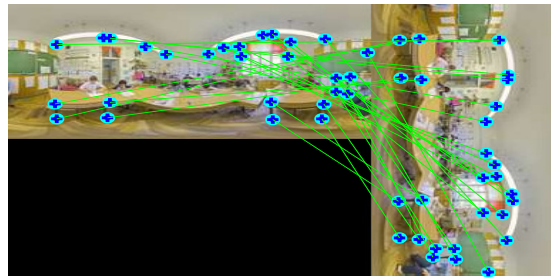

(j)

Fig. 17. Instances of matchings of spherical-spherical $((g),(i),(j))$ and spherical-planar $((a)-$ $(f),(h))$ images of $(a)-(b)$ Desktop, $(c)-(d)$ Parking, $(e)-(h)$ Table and $(i)-(j)$ SUN360 datasets. Green/red lines show correct/incorrect matches respectively and isolated points show no matches. 


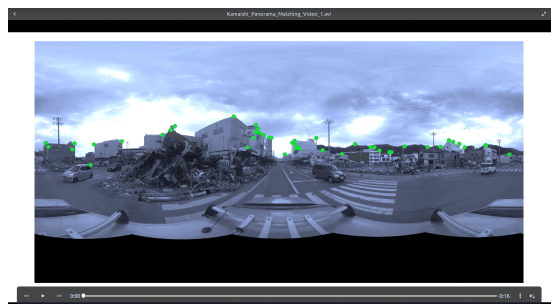

(a)

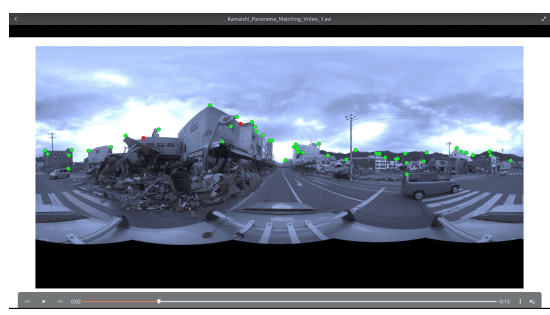

(c)

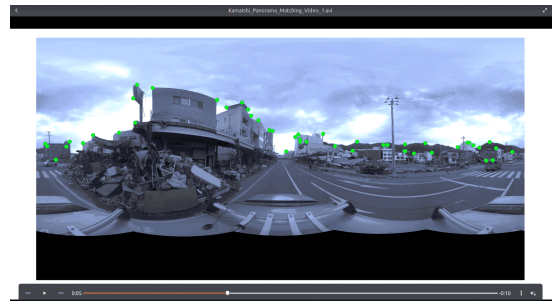

(e)

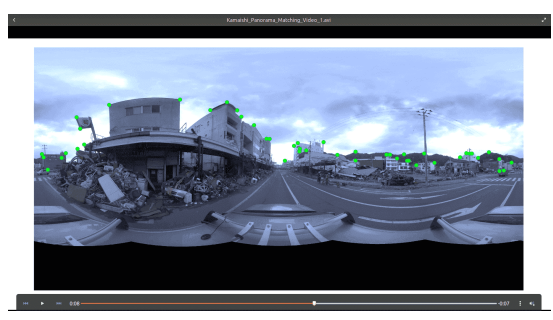

$(\mathrm{g})$

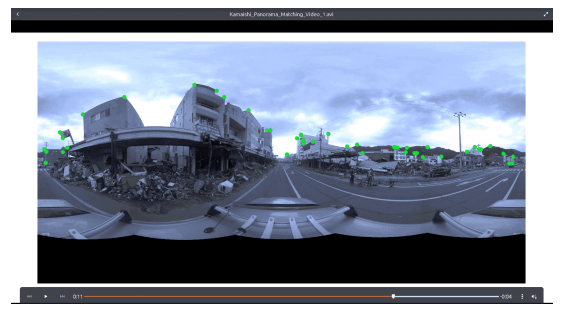

(i)

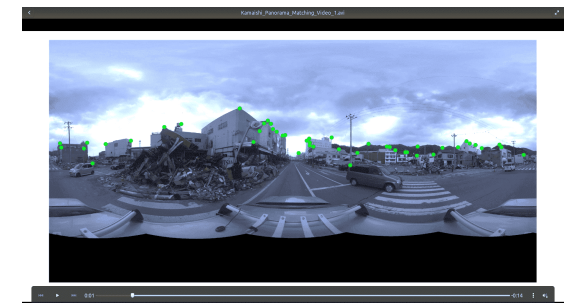

(b)

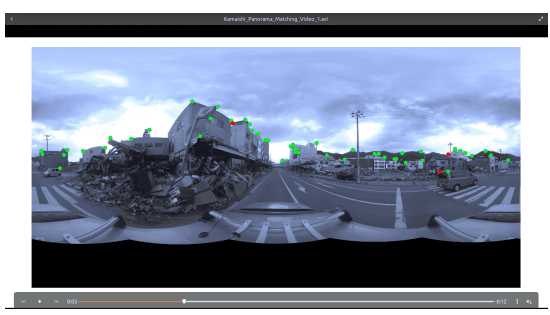

(d)

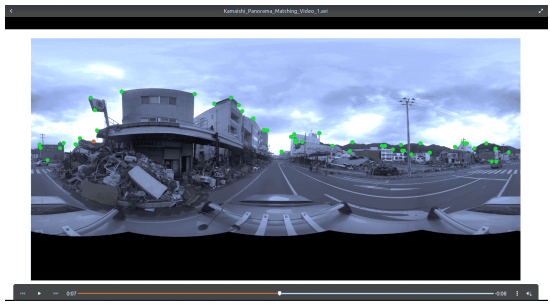

(f)

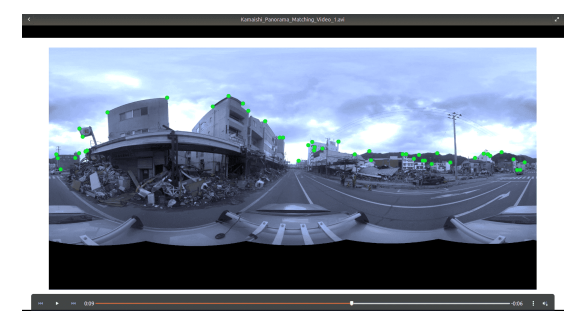

(h)

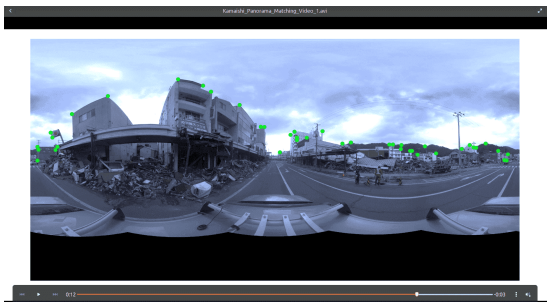

(j)

Fig. 18. Instances of a video of Kamaishi dataset with correct matches as green landmark points and incorrect or zero matches as red landmark points. 